\title{
On a class of growth-maximal hard-core processes
}

\author{
Sven Ebert* and Günter Last $^{\dagger}$
}

November 6, 2017

\begin{abstract}
Generalizing the well-known lilypond model ([4, 11, 2]) we introduce a growthmaximal hard-core model based on a space-time point process $\Psi$ of convex particles. Using a purely deterministic algorithm we prove under fairly general assumptions that the model exists and is uniquely determined by $\Psi$. Under an additional stationarity assumption we show that the model does not percolate. Our model generalizes the lilypond model considerably even if all grains are born at the same time. In that case and under a Poisson assumption we prove a central limit theorem in a large volume scenario.
\end{abstract}

2000 Mathematics Subject Classification. 60G55, 60D05

Key words and phrases. point process, growth model, hard-core model, continuum percolation, lilypond model, central limit theorem

\section{Introduction}

We consider a point process $\Psi=\left\{\left(X_{n}, T_{n}, Z_{n}\right): n \geq 1\right\}$ on $\mathbb{R}^{d} \times \mathbb{R}_{+} \times \mathcal{K}^{d}$, where $\mathbb{R}_{+}:=[0, \infty)$ and $\mathcal{K}^{d}$ denotes the space of all convex bodies containing the origin $0 \in \mathbb{R}^{d}$ in its interior. We interpret $X_{n}$ as the position of a grain (particle) with shape $Z_{n}$ that is born at time $T_{n}$. Without interaction the $n$-th grain starts growing at time $T_{n}$ to form a grain $X_{n}+\left(t-T_{n}\right) Z_{n}:=\left\{X_{n}+\left(t-T_{n}\right) x: x \in Z_{n}\right\}$ at time $t \geq T_{n}$. It ceases its growth as soon as it encounters any other particle. This interaction can occur in two ways. First, $X_{n}$ can be covered by another grain by time $T_{n}$, so that no growth can start at all. Second, the growing grain may touch another grain (which may itself be either growing or have ceased growing at an earlier time). As a result of this growth process a growth time $R_{n} \in \mathbb{R}_{+}$ is attached to the $n$-th particle. The full-grown grain is given by $Z_{n}^{*}:=X_{n}+R_{n} Z_{n}$ if $R_{n}>0$. We use this notation also in case $R_{n}=0$. (Then $Z_{n}^{*}=\left\{X_{n}\right\}$ has empty interior.) The growth protocol (informally) described above entails the following three properties.

(i) The interiors of $Z_{m}^{*}$ and $Z_{n}^{*}$ do not intersect whenever $m \neq n$.

${ }^{*}$ Institut für Stochastik, Karlsruher Institut für Technologie, 76128 Karlsruhe, Germany. Email: sven.ebert@kit.edu

${ }^{\dagger}$ Institut für Stochastik, Karlsruher Institut für Technologie, 76128 Karlsruhe, Germany. Email: guenter.last@kit.edu 
(ii) If $R_{m}=0$ then there is some $n \neq m$ such that $R_{n}>0, X_{m} \in Z_{n}^{*}$ and $T_{m} \geq$ $T_{n}+\inf \left\{r \geq 0: X_{m} \in X_{n}+r Y_{n}\right\}$.

(iii) If $R_{m}>0$ then there is some $n \neq m$ such that $R_{n}>0, T_{m}+R_{m} \geq T_{n}+R_{n}$ and $Z_{m}^{*} \cap Z_{n}^{*} \neq \emptyset$.

Property (i) says that $\left\{Z_{n}^{*}: R_{n}>0\right\}$ is a hard-core system, while (ii) means that the growth of the $n$-th particle is inhibited by some other grain. Property (iii) says that any grain with positive growth time is stopped by some other grain that reached its final size at the same time or earlier. We summarize properties (ii) and (iii) by calling $\left(X_{n}, T_{n}, Z_{n}\right)$ (or $\left.Z_{n}^{*}\right)$ an earlier neighbour of $\left(X_{m}, T_{m}, Z_{m}\right)$ (resp. $Z_{m}^{*}$ ). Any grain must have at least one earlier neighbour. If $\left\{R_{n}: n \geq 1\right\}$ is a family of $\mathbb{R}_{+}$-valued random variables depending measurably on $\Psi$ and such that (i)-(iii) hold almost surely, then we call $\Psi^{*}=\left\{\left(X_{n}, T_{n}, Z_{n}, R_{n}\right): n \geq 1\right\}$ a growth-maximal hard-core model (based on $\Psi$ ). Under certain assumptions on $\Psi$ we will prove in Section 2 that the model exists and is uniquely determined by $\Psi$.
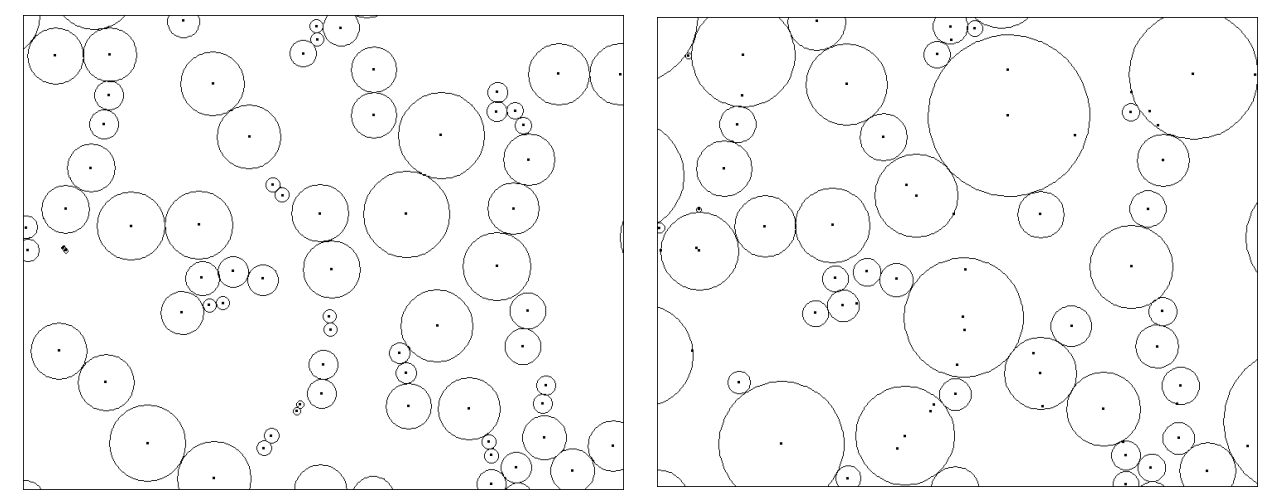

Figure 1: Two models based on the same configuration of positions but with different birth time scenarios.

The lilypond model [4, 11, 2] is a well-known special case of a growth-maximal hardcore model. In this case $\left(T_{n}, Z_{n}\right)=\left(0, B^{d}\right)$ for all $n \geq 1$, where $B^{d}$ is the (closed) unit ball in $\mathbb{R}^{d}$. In the physics literature this was called touch-and-stop model, see [1]. In [5] the model was generalized to the case $\left(T_{n}, Z_{n}\right)=(0, B)$ for a general symmetric star-body $B \subset \mathbb{R}^{d}$. Neither the case of random (possibly different) shapes $Z_{n}$ nor the case of a space-time driving process $\Psi$ has been studied before.

Figure 1 gives an impression of how the introduction of starting times alters the structure of the model. The set of grains is the same in both pictures. But on the left-hand side all birth times are zero while on the right-hand side the birth times are independent and uniformly distributed on the interval $[0,10]$. We see that in the presence of birth times some germs might not be born at all. This in turn implies that the germs which are born have more space to grow. Figure 2 compares the length and cluster size distributions of the preceding two birth time scenarios. The distributions on the righthand side have considerably higher probabilities at large values.

The paper is organized as follows. In Section 2 we use an exteneded version of an algorithm in [2] to prove under fairly general assumptions that the model exists and is 


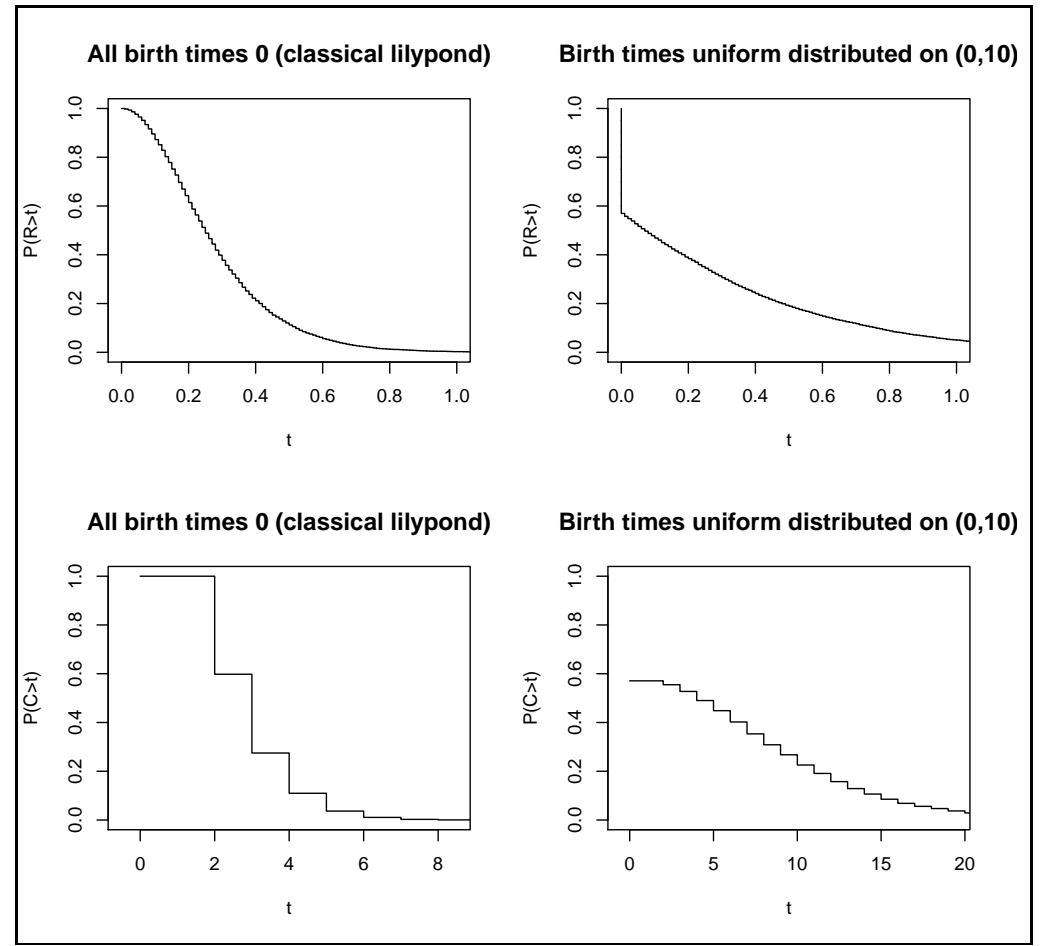

Figure 2: Comparison of length and cluster distributions in different birth time scenarios.

uniquely determined by $\Psi$ in a translation invariant way. In Section 3 we generalize the results in [4, 2] and prove under appropriate stationarity and moment assumptions that there is no percolation in the model. In Section 4 we consider an independently marked stationary Poisson process with constant birth times. Using stabilization arguments as in [9], we prove that the growth times $R_{n}, n \in \mathbb{N}$, satisfy a central limit theorem.

\section{Existence and uniqueness}

Consider $\Psi=\left\{\left(X_{n}, T_{n}, Z_{n}\right): n \geq 1\right\}$, as introduced above, defined on an abstract probability space $(\Omega, \mathcal{F}, \mathbb{P})$. Assume that $\Phi:=\left\{X_{n}: n \geq 1\right\}$ is locally finite, that is $\Phi(B):=\operatorname{card}\left\{m \geq 1: X_{m} \in B\right\}$ is $\mathbb{P}$-a.s. finite for compact sets $B \subset \mathbb{R}^{d}$. For any $k \geq 1$, let $\nu^{(k)}$ denote the $k$-th factorial moment measure of $\Psi$, the measure on $\left(\mathbb{R}^{d} \times \mathbb{R}_{+} \times \mathcal{K}^{d}\right)^{k}$ defined by

$$
\nu^{(k)}(C):=\mathbb{E} \sum^{*} \mathbf{1}\left\{\left(x_{1}, t_{1}, K_{1}, \ldots, x_{k}, t_{k}, K_{k}\right) \in C \cap \Phi^{k}\right\}
$$

for measurable sets $C \subset\left(\mathbb{R}^{d} \times \mathbb{R}_{+} \times \mathcal{K}^{d}\right)^{k}$, where the ${ }^{*}$ indicates that the summation is over all $k$-tuples $\left(\left(x_{1}, t_{1}, K_{1}\right), \ldots,\left(x_{k}, t_{k}, K_{k}\right)\right)$ with pairwise different entries. We shall assume that

$$
\nu^{(k)} \leq a^{k}\left(\lambda^{d} \otimes \mathbb{Q}\right)^{k}, \quad k \geq 1,
$$


where $a>0, \lambda^{d}$ denotes the Lebesgue measure on $\mathbb{R}^{d}$ and $\mathbb{Q}$ a probability measure on $\mathbb{R}_{+} \times \mathcal{K}^{d}$. We shall further suppose that the measure $\mathbb{Q}$ satisfies the integrability condition

$$
\int\left(r^{2 d}+1\right)\left(\rho(K)^{2 d}+1\right) \mathbb{Q}(d(r, K))<\infty
$$

where $\rho(K)$ is the radius of the smallest ball circumscribing $K \in \mathcal{K}^{d}$.

In this section we will prove the following existence and uniqueness result.

Theorem 2.1. Assume that (2.2) and (2.3) hold. Then there exists a unique growthmaximal hard-core model $\Psi^{*}=\left\{\left(X_{n}, T_{n}, Z_{n}, R_{n}\right): n \geq 1\right\}$ based on $\Psi$.

Under the given assumptions the theorem says that there is a family $\left\{R_{n}: n \geq 1\right\}$ of $\Psi$-measurable random variables such that $\Psi^{*}$ satisfies conditions (i)-(iii) given in the introduction almost surely. Moreover, if $\left\{R_{n}^{\prime}: n \geq 1\right\}$ is another such family, then $R_{n}=R_{n}^{\prime} \mathbb{P}$-a.s. for any $n \geq 1$.

Before proving Theorem 2.1 we provide a short discussion of assumption (2.2). For $k \geq 1$ let $\mu^{(k)}$ denote the $k$-th factorial moment measure of $\Phi:=\left\{X_{n}: n \geq 1\right\}$. If $\Psi$ is an independent marking of $\Phi$ with mark distribution $\mathbb{Q}$, then

$$
\nu^{(k)}\left(d\left(x_{1}, t_{1}, K_{1}, \ldots, x_{k}, t_{k}, K_{k}\right)\right)=\mu^{(k)}\left(d\left(x_{1}, \ldots, x_{k}\right)\right) \mathbb{Q}^{k}\left(d\left(t_{1}, K_{1}, \ldots, t_{k}, K_{k}\right)\right)
$$

and (2.2) is implied by $\mu^{(k)} \leq a^{k}\left(\lambda^{d}\right)^{k}, k \geq 1$. The most fundamental example of a point process $\Phi$ with this property is a Poisson process with a bounded intensity function (e.g. a stationary Poisson process). Other examples are provided by certain Cox, Poisson cluster and Gibbs processes, see [2] for more details.

Our proof of Theorem 2.1 is based on a purely deterministic result inspired by Proposition 2.8 in [2]. We start by introducing some notation. Define $\mathbf{N}$ as the set of all countable sets $\psi \subset \mathbb{R}^{d} \times \mathbb{R}_{+} \times \mathcal{K}^{d}$ such that card $\left\{v \in \psi: v \in B \times \mathbb{R}_{+} \times \mathcal{K}^{d}\right\}<\infty$ for all bounded sets $B \subset \mathbb{R}^{d}$ and for all $(x, s, K),(y, t, L) \in \psi$ we have $(x, s, K) \neq(y, t, L)$ only if $x \neq y$. Any $\psi \in \mathbf{N}$ is identified with the (counting) measure card $\{v \in \psi: v \in \cdot\}$ on $\mathbb{R}^{d} \times \mathbb{R}_{+} \times \mathcal{K}^{d}$. A point $(x, s, K) \in \psi$ is interpreted as a grain with shape $K$ located at $x$ and born at time s. As usual we equip $\mathbf{N}$ with the smallest $\sigma$-field $\mathcal{N}$ making the mappings $\psi \mapsto \psi(C)$ measurable for all measurable $C \subset \mathbb{R}^{d} \times \mathbb{R}_{+} \times \mathcal{K}^{d}$. The point process $\Psi$ is then formally defined as a random element in $\mathbf{N}$.

Consider $\psi=\left\{\left(x_{n}, t_{n}, K_{n}\right): n \geq 1\right\} \in \mathbf{N}$. We call a subset $\psi^{*}=\left\{\left(x_{n}, t_{n}, K_{n}, r_{n}\right)\right.$ : $n \geq 1\}$ of $\mathbb{R}^{d} \times \mathbb{R}_{+} \times \mathcal{K}^{d} \times \mathbb{R}_{+}$with projection $\psi$ on $\mathbb{R}^{d} \times \mathbb{R}_{+} \times \mathcal{K}^{d}$ a growth-maximal hard-core model based on $\psi$ if the interiors of $x_{m}+r_{m} K_{m}$ and $x_{n}+r_{n} K_{n}$ do not intersect whenever $m \neq n$ and any point in $\psi^{*}$ has at least one earlier neighbour: If $r_{m}=0$ there is some $n \neq m$ such that $r_{n}>0, x_{m} \in x_{n}+r_{n} K_{n}$ and $t_{m} \geq t_{n}+\inf \left\{r \geq 0: x_{m} \in\right.$ $\left.x_{n}+r K_{n}\right\}$. If $r_{m}>0$ there is some $n \neq m$ such that $r_{n}>0, t_{m}+r_{m} \geq t_{n}+r_{n}$ and $\left(x_{m}+r_{m} K_{m}\right) \cap\left(x_{n}+r_{n} K_{n}\right) \neq \emptyset$. For an earlier neighbour $\left(x_{n}, t_{n}, K_{n}\right)$ of $\left(x_{m}, t_{m}, K_{m}\right)$ we will equivalently use the term stopping neighbour because of the geometric interpretation of our model given in the introduction. Furthermore we will refer to the function $R: \psi \rightarrow \mathbb{R}_{+}$ defined by $R\left(x_{n}, t_{n}, K_{n}\right):=r_{n}, n \in \mathbb{N}$, as a hard-core function on $\psi$ if $\psi^{*}$ is a hard-core model. If $\psi^{*}$ is moreover growth maximal we call $R$ a growth-maximal hard-core function. If $R$ is a hard-core function on $\psi$ we call two different points $u=(x, s, K), v=(y, t, L) \in \psi$ neighbours w.r.t. $R$ if $(x+R(u) K) \cap(y+R(v) L) \neq \emptyset$. 
For convex and non-empty subsets $K, K^{\prime}, L, L^{\prime}$ of $\mathbb{R}^{d}$ we define

$$
a\left(K, K^{\prime}, L, L^{\prime}\right):=\inf \left\{r \geq 0:\left(K+r K^{\prime}\right) \cap\left(L+r L^{\prime}\right) \neq \emptyset\right\}
$$

and use abbreviations of the type $a(x, K, y, L):=a(\{x\}, K,\{y\}, L), x, y \in \mathbb{R}^{d}$. For two grains $u:=(x, s, K)$ and $v:=(y, t, L)$ we define the first contact time $d(u, v)$ as follows. If $s \leq t$ we set

$$
\begin{aligned}
d(u, v):= & \mathbf{1}\{a(x, K, y, 0) \leq t-s\}(s+a(x, K, y, 0)) \\
& +\mathbf{1}\{a(x, K, y, 0)>t-s\}(t+a(x+(t-s) K, K, y, L)) .
\end{aligned}
$$

In the case covered by the first summand, the growing grain $u$ reaches the point $y$ not later than the birth time $t$ of grain $v$. In the second case both grains start to grow and meet at time $d(u, v)$. If $s \geq t$ we define $d(u, v):=d(v, u)$, so that $d(\cdot, \cdot)$ becomes symmetric. In the following and later we write $a \wedge b:=\min \{a, b\}$ and $a \vee b:=\max \{a, b\}$ for $a, b \in \mathbb{R}$.

Lemma 2.2. Let $R$ be a hard-core function on $\psi \in \mathbf{N}$. In addition suppose $u=(x, s, K)$ and $v=(y, t, L)$ to be neighbours in $\psi$ w.r.t. $R$ and $R(u) \wedge R(v)>0$. Then

$$
(R(u)+s) \wedge(R(v)+t) \leq d(u, v) \leq(R(u)+s) \vee(R(v)+t)
$$

Here the second inequality is strict, provided that $R(u)+s \neq R(v)+t$.

Proof: We assume w.l.o.g. $s \leq t$ and prove the assertion by contradiction. First suppose that $(R(u)+s) \wedge(R(v)+t)>d(u, v)$. If $a(x, K, y, 0) \leq t-s$ then

$$
R(u)+s>d(u, v)=s+a(x, K, y, 0),
$$

where we have used (2.5) to get the identity. Since $y \in x+a(x, K, y, 0) K$, this contradicts $R(v)>0$ and the hard-core property. If $a(x, K, y, 0)>t-s$, then $d(u, v)=t+r$, where $r:=a(x+(t-s) K, K, y, L)$. Therefore $R(v)>r$. But the definition of $a(\cdot)$ implies $(x+(t-s+r) K) \cap(y+r L) \neq \emptyset$ and hence $R(u)<t-s+r=d(u, v)-s$, by the hard-core property. This contradicts our assumption $R(u)+s>d(u, v)$.

Now suppose $(R(u)+s) \vee(R(v)+t)<d(u, v)$. If $a(x, K, y, 0) \leq t-s$, then

$$
R(v)+t<d(u, v)=s+a(x, K, y, 0) \leq t,
$$

contradicting $R(v)>0$. If $a(x, K, y, 0)>t-s$ we obtain

$$
R(v)+t<d(u, v)=t+a(x+(t-s) K, K, y, L)=t+r,
$$

so that the definition of $a(\cdot)$ implies $(x+(t-s+r) K) \cap(y+R(v) L)=\emptyset$. Since $u$ and $v$ are neighbours we get $R(u)>t-s+r=d(u, v)-s$, contradicting our assumption $R(u)+s<d(u, v)$.

To prove the final assertion we again argue by contradiction. As the case $a(x, K, y, 0) \leq$ $t-s$ can be treated as above we assume that $a(x, K, y, 0)>t-s$. Assume first that $d(u, v) \geq R(u)+s>R(v)+t$. As before we then get $R(u)>d(u, v)-s$, a contradiction. Let us finally assume that $d(u, v) \geq R(v)+t>R(u)+s$. Then the definition of $a(\cdot, \cdot)$ implies that $R(u) \geq t-s+r=d(u, v)-s$, contradicting $d(u, v)>R(u)+s$. 
The translation of $\psi \in \mathbf{N}$ by $y \in \mathbb{R}^{d}$ is defined by $y+\psi:=\{(y+x, s, K):(x, s, K) \in \psi\}$. A set $H \subset \mathbf{N}$ is translation invariant if $y+\psi \in H$ for all $\psi \in H$ and all $y \in \mathbb{R}^{d}$. For a mapping $R: \mathbf{N} \times \mathbb{R}^{d} \times \mathbb{R}_{+} \times \mathcal{K}^{d} \rightarrow \mathbb{R}_{+}$and $\psi \in \mathbf{N}$ we define $R^{\dagger}(\psi) \subset \mathbb{R}^{d} \times \mathbb{R}_{+} \times \mathcal{K}^{d} \times \mathbb{R}_{+}$ by

$$
R^{\dagger}(\psi):=\{(x, t, K, R(\psi, x, s, K)):(x, s, K) \in \psi\}
$$

If $R$ is translation invariant in the sense that

$$
R(y+\psi, x+y, t, K)=R(\psi, x, s, K), \quad \psi \in \mathbf{N}, x, y \in \mathbb{R}^{d},(s, K) \in \mathbb{R}_{+} \times \mathcal{K}^{d},
$$

then $R^{\dagger}(y+\psi)=R^{\dagger}(\psi)$ for all $(\psi, y) \in \mathbf{N} \times \mathbb{R}^{d}$.

Theorem 2.1 is implied by the following Proposition and Lemma 2.4.

Proposition 2.3. There exist a measurable mapping $R: \mathbf{N} \times \mathbb{R}^{d} \times \mathbb{R}_{+} \times \mathcal{K}^{d} \rightarrow \mathbb{R}_{+}$and a set $H \in \mathcal{N}$ such that if $\psi \in H$ then $R^{\dagger}(\psi)$ is the unique growth-maximal hard-core model based on $\psi$. Moreover $H$ and $R$ are translation invariant.

The next lemma shows that $H$ is reasonably big.

Lemma 2.4. Let $H$ be the set from Proposition 2.3. Then $\mathbb{P}(\Psi \in H)=1$.

Proof of Proposition 2.3: Our strategy for the proof is as follows. We first construct $H$ and $R$ via a recursive algorithm. Growth-maximality and the uniqueness property will be proved in two separate lemmas.

Take $\psi \in \mathbf{N}$. Starting with $\psi_{0}:=\emptyset$ we will recursively construct an increasing sequence $\psi_{i} \subset \psi, i \in \mathbb{N}$, together with a sequence of hard-core functions $R_{i}: \psi_{i} \rightarrow \mathbb{R}_{+}$such that $R_{i+1}$ extends $R_{i}$ for any $i \geq 0$. We will further define $R(\psi, u):=R_{i}(u)$ whenever $u \in \psi_{i}$ for some $i$. Afterwards this definition will be extended to all other $u \in \psi$ in a suitable way.

Let $i \in \mathbb{N}$. For $u \in \psi \backslash \psi_{i}$ we define

$$
\begin{gathered}
\nu_{i}(u):=\{u\} \cup\left\{v \in \psi \backslash\left(\psi_{i} \cup\{u\}\right): v \text { and } u\right. \text { are mutual nearest neighbours } \\
\text { in } \left.\psi \backslash \psi_{i} \text { with respect to } d\right\}
\end{gathered}
$$

We assume from now on that $\psi$ satisfies

$$
d(u, v) \neq d\left(u^{\prime}, v^{\prime}\right), \quad u, u^{\prime}, v, v^{\prime} \in \psi,\{u, v\} \neq\left\{u^{\prime}, v^{\prime}\right\} .
$$

Then $\nu_{i}(u)$ contains at most two points. Furthermore let

$$
\varphi_{i}:=\left\{u \in \psi \backslash \psi_{i}: \nu_{i}(u) \neq\{u\}\right\}
$$

be the set of all mutual nearest neighbours in $\psi \backslash \psi_{i}$ (w.r.t. $d$ ). In the case $\varphi_{i}=\emptyset$ we define $\psi_{i+1}=\psi_{i}$ i.e. $\psi_{j}=\psi_{i}, j \geq i$. Otherwise we obtain $\psi_{i+1}$ based on $\psi_{i}$ in the following way. Set $f(u, v):=\inf \left\{r \geq 0:(x+r K) \cap\left(y+R_{i}(v) L\right) \neq \emptyset\right\}+s$ for $u=(x, s, K) \in \psi \backslash \psi_{i}$ and $v=(y, t, L) \in \psi_{i}$. For $u \in \varphi_{i}$ define $d_{i}(u):=\inf _{v \in \psi_{i}, R_{i}(v)>0} f(u, v)$ as well as

$$
s_{i}^{\prime}(u):=d(u, v), v \in \nu_{n}(u) \backslash\{u\} \quad \text { and } \quad s_{i}^{\prime \prime}(u):=\min _{v \in \nu_{i}(u)} d_{i}(v) .
$$


Now we add points of $\varphi_{i}$ to $\psi_{i}$ by the following procedure to obtain $\psi_{i+1}$.

Consider $u=(x, s, K) \in \varphi_{i}$ so that there exists $v=(y, t, L) \in \nu_{i}(u) \backslash\{u\}$. Assume first that $s_{i}^{\prime}(u) \leq s_{i}^{\prime \prime}(u)$. If $s \leq t$ and $a(x, K, y,\{0\}) \leq t-s$ we set $R_{i+1}(v):=0$ and add $v$ to $\psi_{i}$. If $s \leq t$ and $a(x, K, y,\{0\})>t-s$ we add $u$ and $v$ to $\varphi_{i}$ and define $R_{i+1}(u):=s_{i}^{\prime}(u)-s$ and $R_{i+1}(v):=s_{i}^{\prime}(u)-t$. In case $t \leq s$ we apply the same rules as before with the roles of $u$ and $v$ interchanged. Now we assume $s_{i}^{\prime \prime}(u)<s_{i}^{\prime}(u)$. For all $w:=(z, r, M)$ with $w \in \nu_{i}(u)$ and $d_{i}(w)=s_{i}^{\prime \prime}(u)$, we set $R_{i+1}(w):=s_{i}^{\prime \prime}(u)-r$ and add those points to $\psi_{i}$. Applying this algorithm to every $u \in \varphi_{i}$ yields the $(i+1)$-th hard-core function $R_{i+1}$ on $\psi_{i+1}$.

We define

$$
\psi_{\infty}:=\bigcup_{i=1}^{\infty} \psi_{i} \quad \text { and } \quad \varphi:=\psi \backslash \psi_{\infty}=\bigcap_{i=1}^{\infty} \psi \backslash \psi_{i} .
$$

Set $R(\psi, u):=R_{i}(u)$ whenever $u \in \psi_{i}$ for some $i$. If $\operatorname{card} \varphi=1$ and $\left\{v \in \psi_{\infty}: R(v)>\right.$ $0\} \neq \emptyset$ we define $R(\psi, u):=\inf _{v \in \psi_{\infty}, R(\psi, v)>0} f(u, v)-s$ for $u=(x, s, K) \in \varphi$.

Let $H$ denote the set of all $\psi$ satisfying (2.7) and for which the above algorithm yields a set $\psi_{\infty}$ with $\operatorname{card}\left(\psi \backslash \psi_{\infty}\right) \leq 1$ and $\left\{v \in \psi_{\infty}: R(\psi, v)>0\right\} \neq \emptyset$. For all $\psi \in \mathbf{N} \backslash H$, we set $R(\psi, \cdot) \equiv 0$. Then $R$ is a hard-core function. By construction the set $H$ is translation invariant, and $R$ is translation-invariant on $H$. Thus $R$ is translation-invariant. We need to show that $R(\psi, \cdot)$ is for any $\psi \in H$ the unique growth-maximal hard-core function on $\psi$. To aid reading, we do this in two separate Lemmas.

Lemma 2.5. Assume that $\psi \in H$. Then $R:=R(\psi, \cdot)$ is a growth-maximal function.

Proof: Let $u=(x, s, K) \in \psi$. To show that $u$ has an earlier neighbour. We distinguish the cases $R(u)=0$ and $R(u)>0$.

1. Assume that $R(u)=0$. By the algorithm (introduced in the above first part of the proof of Proposition 2.3) there is an $u \in \psi_{\infty}$, that is $u \in \psi_{i+1} \backslash \psi_{i}$ for some $i \geq 0$. Moreover, the algorithm implies that there exists a $v=(y, t, L) \in \psi \backslash\{u\}$ such that $\{u, v\}=\nu_{i}(u)$ and

$$
s_{i}^{\prime}(v) \leq s_{i}^{\prime \prime}(v) .
$$

We show, that $v$ is an earlier neighbour of $u$. First we assume that $v \in \psi_{j+1} \backslash \psi_{j}$ for some $j>i$ (the other case being $\left.v \in \psi \backslash \psi_{\infty}\right)$. Thus there is a $w=(z, r, M) \in \psi \backslash\left(\psi_{j} \cup\{u, v\}\right)$ with $\{v, w\}=\nu_{j}(v)$.

Assume now that $s_{j}^{\prime}(v) \leq s_{j}^{\prime \prime}(v)$. Since $u, v$ are mutual nearest neighbours in $\psi \backslash \psi_{i} \supset$ $\psi \backslash \psi_{i}$ and (2.7) holds (since $\psi \in H$ ), we have

$$
d(v, w)>d(v, u) .
$$

We now claim $R(v)>0$. Assuming on the contrary that $R(v)=0$ we obtain from $R(u)=0$ and (2.5) that $t \leq s$,

$$
a(x, 0, y, L)=\leq s-t
$$

and

$$
t+a(x, 0, y, L)=d(u, v)
$$


Using the same argument for the pair $(v, w)$ we obtain

$$
t+a(x, 0, y, L)=d(u, v)<d(w, v)=r+a(y, 0, z, M)
$$

and, moreover, $a(y, 0, z, M) \leq t-r$. It follows that $t+a(x, 0, y, L)<t$, a contradiction. Recalling $s_{j}^{\prime}(v) \leq s_{j}^{\prime \prime}(v)$ we derive from $R(v)>0$ the first equality in

$$
R(v)=d(v, w)-t>d(v, u)-t=a(x, 0, y, L)
$$

and therefore

$$
x \in y+R(v) L .
$$

Together with (2.12) this implies that $v$ is indeed an earlier neighbour of $u$.

Assume now that $s_{j}^{\prime}(v)>s_{j}^{\prime \prime}(v)$. By the algorithm there exists a neighbour $w^{\prime}:=$ $\left(z^{\prime}, r^{\prime}, M^{\prime}\right) \in \psi_{j}$ of $v$ with $R\left(w^{\prime}\right)>0$. Further there are $k<j$ and $w^{\prime \prime}=\left(z^{\prime \prime}, r^{\prime \prime}, M^{\prime \prime}\right)$, such that $w^{\prime}$ and $w^{\prime \prime}$ are mutual nearest neighbours in $\psi \backslash \psi_{k}$. Hence the algorithm implies the first identity in

$$
R\left(w^{\prime}\right)+r^{\prime}=s_{k}^{\prime}\left(w^{\prime}\right) \wedge s_{k}^{\prime \prime}\left(w^{\prime}\right) \leq s_{k}^{\prime}\left(w^{\prime}\right)=d\left(w^{\prime}, w^{\prime \prime}\right) \leq d\left(w^{\prime}, v\right),
$$

where the final inequality comes from the fact that $w^{\prime}, w^{\prime \prime}$ are mutual nearest neighbours in $\psi \backslash \psi_{k} \supset \psi \backslash \psi_{j}$. Since $w^{\prime}$ and $v$ are neighbours, Lemma 2.2 yields

$$
R(v)+t \geq d\left(v, w^{\prime}\right) .
$$

Again we have to distinguish two subcases. If $k \geq i$ we get from the fact that $u$ and $v$ are mutual nearest neighbours in $\psi \backslash \psi_{i} \supset \psi \backslash \psi_{k}$ that $d\left(w^{\prime}, v\right) \geq d(u, v)$. Together with (2.15) and (2.13) this yields

$$
R(v) \geq d\left(v, w^{\prime}\right)-t \geq d(u, v)-t=a(x, 0, y, L) .
$$

Hence (2.14) follows. Assume now that $k<i$. To show that

$$
R(v)=a\left(y, L, z^{\prime}+R\left(w^{\prime}\right) M^{\prime}, 0\right) \geq a(x, 0, y, L),
$$

we assume on the contrary that the inequality fails. But then the algorithm implies $s_{i}^{\prime}(v)>s_{i}^{\prime \prime}(v)$, contradicting (2.10).

Now we assume that $v \in \psi \backslash \psi_{\infty}$. To show that $v$ is an earlier neighbour of $u$ we can proceed analogously to the case $s_{j}^{\prime}(v)>s_{j}^{\prime \prime}(v)$ from above.

2. Assume that $R(u)>0$. We have to consider two subcases. If $s_{i}^{\prime}(u) \leq s_{i}^{\prime \prime}(u)$ there exists $v=(y, t, L) \in \psi$ such that $\{u, v\}=\nu_{i}(u), R(u)=d(u, v)-s>0$ and $R(v)=d(u, v)-t>0$. Therefore $R(u)+s=R(v)+t$ and $v$ is an earlier neighbour of $u$. If $s_{i}^{\prime}(u)>s_{i}^{\prime \prime}(u)$ then $i \geq 1$ and there exists a neighbour $v=(y, t, L) \in \psi_{i}$ of $u$ with $R(v)>0$. We show $R(u)+s \geq R(v)+t$ by assuming on the contrary that $R(u)+s<R(v)+t$. There exist a $k \in\{0, \ldots, i-1\}$ and a $w$ with $v \in \psi_{k+1} \backslash \psi_{k}$ and $\{v, w\}=\nu_{i}(v)$. The algorithm implies that $R(v)+s \leq d(v, w)$. Lemma 2.2 and our assumption $R(u)+s<R(v)+t$ yield $R(u)+s>d(u, v)$. This gives $d(u, v)<d(v, w)$ which contradicts the mutual nearest neighbour property of $v$ and $w$ in $\psi \backslash \psi_{k-1}$.

In the remaining case $u \in \psi \backslash \psi_{\infty}$ we can proceed as in the second case of the last paragraph. Thus every $u \in \psi$ has at least one earlier neighbour. 
Lemma 2.6. Assume that $\psi \in H$. Then $R:=R(\psi, \cdot)$ is the unique growth-maximal function on $\psi$.

ProOF: Let $R^{\prime}$ be some growth-maximal hard-core function on $\psi$. First we prove by by induction over the steps of the algorithm that $R(u)=R^{\prime}(u)$ whenever $u \in \psi_{\infty}$ satisfies $R(u)>0$.

Let $u=(x, s, K) \in \psi_{1}$ satisfy $R(u)>0$. The then yields algorithm that

$$
d(u, v)=R(u)+s=R(v)+t,
$$

where $v=(y, t, L)$ is the mutually nearest neighbour of $u$ in $\psi$. Assume $R^{\prime}(u)=0$. Then there exists $w=(z, r, M) \in \psi \backslash\{u\}$ with $R^{\prime}(w) \geq a(z, M, x, 0)$ and $s-r>a(z, M, x, 0)$. Hence

$$
d(u, w)=a(z, M, x, 0)+r<s .
$$

If $s \leq t$, we get $d(u, v)=t+a(x+(t-s) K, K, y, L)>s$. If $t<s$, we also get $d(u, v)=s+a(x, K, y+(s-t) L, L)>s$. In view of (2.17) we obtain that $w \neq v$ and $d(u, w)<d(u, v)$. But $w \neq v$ leads to $d(u, w)<d(u, v)$. As this contradicts the mutual nearest neighbour property of $u$ and $v$, we must have that $R^{\prime}(u)>0$.

Assume that $R(u) \neq R^{\prime}(u)$. By Lemma 2.2 and (2.16) we can assume w.l.o.g. that

$$
R^{\prime}(u)+s<d(u, v) .
$$

Let $w \in \psi$ be a stopping neighbour of $u$ w.r.t. $R^{\prime}$. In particular, $R^{\prime}(w)>0$. By Lemma 2.2 we conclude $R^{\prime}(u)+s \geq d(u, w)$. Hence (2.18) implies that $d(u, w)<d(u, v)$, a contradiction.

Now we take $u=(x, s, K) \in \psi_{i+1} \backslash \psi_{i}$ for some $i \geq 1$ and assume that $R(u)>0$. Again we first show that $R^{\prime}(u)>0$. Assume $R^{\prime}(u)=0$. Then there exists $w \in \psi \backslash\{u\}$ with $R^{\prime}(w) \geq a(z, M, x, 0)>0$ and $s-r \geq a(z, M, x, 0)$. Assume $w \in \psi \backslash \psi_{i}$. Then we can argue as in the case $u \in \psi_{1}$ to conclude that $R^{\prime}(u)>0$. Hence we can assume that $w \in \psi_{i}$. from the induction hypothesis we get $R^{\prime}(w)=R(w)$. Therefore,

$$
d(w, u)=a(z, M, x, 0)+r<R(w)+r .
$$

Due to the algorithm $0<R(w)+r \leq d\left(w, w^{\prime}\right)$, where $w^{\prime}$ is a mutually nearest neighbour (w.r.t. $R$ ) of $w$ in $\psi \backslash \psi_{j}$ for some $j \leq i-1$. Therefore $d(w, u) \leq d\left(w, w^{\prime}\right)$ contradicting the choice of $w^{\prime} \in \psi \backslash \psi_{j} \supset \psi \backslash \psi_{i}$ and the fact that $u \in \psi \backslash \psi_{i}$. Thus $R^{\prime}(u)>0$.

Next we show that $R(u)=R^{\prime}(u)$. Assume $\nu_{i}(u)=\{u, v\}$ and

$$
R(u)=s_{i}^{\prime}(u)-s=d(u, v)-s .
$$

Assume moreover that $R^{\prime}(u)<s_{i}^{\prime}(u)-s$. Then we can argue as at (2.18) to conclude that $u$ cannot have an earlier neighbour in $\psi \backslash \psi_{i}$ w.r.t. $R^{\prime}$. Hence there exists an earlier neighbour $w \in \psi_{i}$ of $u$ w.r.t. $R^{\prime}$, that is $R^{\prime}(w)+r \leq R^{\prime}(u)+s$ and $R^{\prime}(w)>0$. By induction hypothesis $R(w)=R^{\prime}(w)$. Therefore the algorithm implies

$$
f(u, w)=\inf \{r \geq 0:(x+r K) \cap(z+R(w) M) \neq \emptyset\}+s=R^{\prime}(u)+s<s_{i}^{\prime}(u) .
$$


This leads to

$$
f(u, w)<s_{i}^{\prime}(u) \leq s_{i}^{\prime \prime}(u)=\inf _{v \in \psi_{i}, R(v)>0} f(u, v) \leq f(u, w)<s_{i}^{\prime}(u),
$$

a contradiction. Now assume $R^{\prime}(u)>s_{i}^{\prime}(u)-s$. The hard-core property of $R^{\prime}$ yields that $R^{\prime}(v)<s_{i}(v)-t$. But we have just proved that this is not possible. Hence only the desired case $R^{\prime}(u)=s_{i}^{\prime}(u)-s=R(u)$ remains.

Now assume $R(u)+s=s_{i}^{\prime \prime}(u)<s_{i}^{\prime}(u)$. Then there exists $v \in \psi_{i}$ with $R(v)>0$ and $s_{i}^{\prime \prime}(u)=f(u, v)+s$. By induction hypothesis $R(v)=R^{\prime}(v)$. Hence the hard-core property of $R^{\prime}$ that

$$
s_{i}^{\prime \prime}(u)+s=f(u, v) \geq R^{\prime}(u) .
$$

To show that $R(u)=R^{\prime}(u)$ we assume on the contrary that the above inequality is strict. Since, by induction hypothesis, $R(w)=R^{\prime}(w)$ for all $w \in \psi_{i}$ with $R(w)>0$, we obtain from $s_{i}^{\prime \prime}(u)+s=f(u, v)$ and the definition of $f(u, v)$ that $u$ has no neighbour in $\psi_{i}$ w.r.t. $R^{\prime}$. Because of $s_{i}^{\prime \prime}(u)<s_{i}^{\prime}(u)$ we get that $0<R^{\prime}(u)+s<f(u, v)<d\left(u, v^{\prime}\right)$ where $v^{\prime}$ is the mutual nearest neighbour of $u$ in $\psi_{i+1} \backslash \psi_{i}$. By Lemma 2.2 we deduce that

$$
R^{\prime}(w)+r \leq d(u, w)>d\left(u, v^{\prime}\right)>R^{\prime}(u)+s,
$$

for every neighbour $w=(z, r, M) \in \psi \backslash \psi_{i}$ of $u$. Therefore $U$ cannot have an earlier neighbour in $\psi \backslash \psi_{i}$ w.r.t. $R^{\prime}$. This is a contradiction.

Next we show $R(u)=R^{\prime}(u)$ for $u \in \psi \backslash \psi_{\infty}$. Due to the algorithm we can assume $R(u)>0$. Because of $R(v)=R^{\prime}(v)$ for all $v \in \psi_{\infty}$ with $R(v)>0$ and the hard-core property of $R^{\prime}$ we obtain the inequality $R^{\prime}(u) \leq \inf _{v \in \psi_{\infty}, R(v)>0} f(u, v)-s=R(u)$. If $R^{\prime}(u)<R(u)$ the point $u$ would not have an earlier neighbour by the definition of $f(u, v)$.

Finally we show that $R^{\prime}(u)=0$ holds for all $u \in \psi$ with $R(u)=0$. For $u \in \psi$ with $R(u)=0$ let $v \in \psi$ be an earlier neighbour of $u$ in $\psi$ w.r.t. $R$. Therefore it follows $R(v)>0$ and $x \in R(v) L$. We have already shown above $R^{\prime}(v)=R(v)$. Thus $x \in R^{\prime}(v) L$. Since $R^{\prime}$ is a hard-core function this yields $R^{\prime}(u)=0$.

For a Borel set $A \subset \mathbb{R}^{d} V_{d}(A)$ the volume of $A$. Further let $\kappa_{d}:=V_{d}\left(B^{d}\right)$ denote the volume of the unit ball. The proof of Lemma 2.4 uses the following lemma.

Lemma 2.7. Let $r \geq 0,0 \leq s \leq t$ and $K_{1}, K_{2} \in \mathcal{K}^{d}$ with $K_{1} \subset x_{1}+r_{1} B^{d}, K_{2} \subset x_{2}+r_{2} B^{d}$ for some $r_{1}, r_{2} \geq 0$ and $x_{1}, x_{2} \in \mathbb{R}^{d}$. Then

$$
V_{d}\left(r K_{1}+t K_{2}\right)-V_{d}\left(r K_{1}+s K_{2}\right) \leq \max _{i=1, \ldots, d}\left(t^{i}-s^{i}\right) \sum_{j=0}^{d-1} \kappa_{d}\left(\begin{array}{l}
d \\
j
\end{array}\right) r^{j} r_{1}^{j} r_{2}^{d-j}
$$

ProOf: Using mixed volumes (see e.g. [12, (5.1.26)]), we obtain

$$
V_{d}\left(r K_{1}+t K_{2}\right)=\sum_{j=0}^{d}\left(\begin{array}{l}
d \\
j
\end{array}\right) r^{j} t^{d-j} V\left(K_{1}[j], K_{2}[d-j]\right)
$$

where we have also used the homogeneity part of [12, (5.1.24)]. Therefore

$$
V_{d}\left(r K_{1}+t K_{2}\right)-V_{d}\left(r K_{1}+s K_{2}\right)=\sum_{j=0}^{d-1}\left(\begin{array}{l}
d \\
j
\end{array}\right) r^{j}\left(t^{d-j}-s^{d-j}\right) V\left(K_{1}[j], K_{2}[d-j]\right) .
$$


Since $K_{i} \subset x_{i}+r_{i} B^{d}$ we can now use the monotonicity property [12, (5.1.23)] and the translation invariance of mixed volumes to obtain that

$$
V_{d}\left(r K_{1}+t K_{2}\right)-V_{d}\left(r K_{1}+s K_{2}\right) \leq \sum_{j=0}^{d-1}\left(\begin{array}{l}
d \\
j
\end{array}\right) r^{j}\left(t^{d-j}-s^{d-j}\right) r_{1}^{j} r_{2}^{d-j} V_{d}\left(B^{d}\right),
$$

where we have used that $V\left(B^{d}, \ldots, B^{d}\right)=V_{d}\left(B^{d}\right)$. This implies the assertion.

Proof of Lemma 2.4: We construct a measurable set $G \subset H$ for which we can show that $\mathbb{P}(\Psi \in G)=1$. Consider an increasing sequence $b_{i}>0, i \in \mathbb{N}_{0}$, with $\lim _{i \rightarrow \infty} b_{i}=\infty$ to be specified later and define $W_{j}:=[-j, j]^{d}, j \geq 1$. Moreover for $n \in \mathbb{N}, s<t$ and a bounded Borel set $B \subset \mathbb{R}^{d}$ the set $F_{n}(s, t, B)$ is defined as follows. A set $\psi \in$ $\mathbf{N}$ belongs to $F_{n}(s, t, B)$, if it contains $n+1$ different points $u_{0}, \ldots, u_{n}$ such that $s<$ $d\left(u_{0}, u_{1}\right), \ldots, d\left(u_{n-1}, u_{n}\right) \leq t$ and $u_{0} \in B^{\prime}:=B \times \mathbb{R}_{+} \times \mathcal{K}^{d}$. Define

$$
F(s, t, B):=\bigcap_{n \geq 1} F_{n}(s, t, B)
$$

and $G$ as follows. A set $\psi \in \mathbf{N}$ belongs to $G$ if it satisfies (2.7) and

$$
\begin{aligned}
& \operatorname{card}\{v \in \psi \backslash\{u\}: d(u, v) \leq r\}<\infty, \quad r>0, u \in \psi, \\
& a(x, K, y, L) \neq|s-t|, \quad(x, s, K),(y, t, L) \in \psi, x \neq y
\end{aligned}
$$

as well as $\psi \notin F$ where

$$
F:=\bigcup_{i=0}^{\infty} \bigcup_{j=1}^{\infty} F\left(b_{i}, b_{i+1}, W_{j}\right)
$$

We recall the definition of the set $H$ from the proof of Proposition 2.3 as the set of all $\psi \in \mathbf{N}$ which satisfy (2.7) and the algorithm of Proposition 2.3 yields (based on $\psi$ ) a set $\psi_{\infty}$ with $\operatorname{card}\left(\psi \backslash \psi_{\infty}\right) \leq 1$ and $\left\{v \in \psi_{\infty}: R(\psi, v)>0\right\} \neq \emptyset$. We now show $G \subset H$ an take $\psi \in G$. Define $\varphi:=\psi \backslash \psi_{\infty}$ and assume card $\varphi \geq 2$. Furthermore we take $u \in \varphi$ and define $\nu(u)$ as the set of all mutual nearest neighbours of $u$ in $\varphi$ and set $\nu(u)=\{v\}$. Define $A(u):=\{w \in \psi: d(u, w) \leq d(u, v)\} \cup\{w \in \psi: d(v, w) \leq d(v, u)\}$. By construction $A(u)$ contains no points of $\varphi \backslash(\nu(u) \cup\{u\})$. Because of (2.21) the set $A(u)$ is finite. So it contains no points of $\psi_{\infty}$, since the corresponding growth times would have been determined until the $i$-th step of the algorithm for some $i \in \mathbb{N}_{0}$. Since a pair of mutual nearest neighbours $u$ and $v$ in $\varphi$ would also be a pair of mutual nearest neighbours in $\psi \backslash \psi_{i}$ at least one point of $\varphi$ would have attained a growth time, which is a contradiction to the definition of $\varphi$. In conclusion $\nu(u)$ has to be empty. By induction and the symmetry of $d$ we show that in this case $\psi$ must have a descending chain, i.e. an infinite sequence $u_{1}, u_{2}, \ldots$ of pairwise different points of $\psi$ with $d\left(u_{n}, u_{n+1}\right) \leq d\left(u_{n-1}, u_{n}\right)$ for any $n \geq 2$ (see also [4] and [2]). Since $\varphi$ contains no pair of mutual nearest neighbours there exist three different points $u_{1}, u_{2}, u_{3}$ with $d\left(u_{1}, u_{2}\right) \geq d\left(u_{2}, u_{3}\right)$ and $u_{3}$ being the nearest neighbour of $u_{2}$ and $u_{2}$ the nearest neighbour of $u_{1}$. Inductively suppose $u_{1}, \ldots, u_{n}$, $n>2$, to be $n$ different points in $\varphi$ with $u_{i}$ being a nearest neighbour of $u_{i-1}$ satisfying 
$d\left(u_{i}, u_{u-1}\right) \leq d\left(u_{i-1}, u_{i-2}\right), i=3, \ldots, n$. Let $u_{n+1}$ be a nearest neighbour of $u_{n}$. This implies $d\left(u_{n+1}, u_{n}\right) \leq d\left(u_{n}, u_{n-1}\right)$ since otherwise, by the symmetry of $d$, we would get a contradiction to the nearest neighbour property. To show $u_{n-1} \notin\left\{u_{1}, \ldots, u_{n}\right\}$ we assume $u_{n+1}=u_{j}$ for some $j \in\{1, \ldots, n-1\}$. If $u_{n}$ would not be a nearest neighbour of $u_{j}$ we get for some $u \in \varphi$

$$
d\left(u_{j}, u\right)<d\left(u_{j}, u_{n}\right)=d\left(u_{n+1}, u_{n}\right) \leq d\left(u_{j+1}, u_{j}\right)=d\left(u_{j}, u_{j+1}\right) .
$$

Since $u_{j+1}$ is a nearest neighbour of $u_{j}$ this is a contradiction and $u_{n}$ has to be a nearest neighbour of $u_{j}$. But this leads to $u_{n+1}$ and $u_{n}$ being mutual nearest neighbours contrary to our assumption. Hence $\varphi$ would contain a descending chain and so $\psi$ would also contain a descending chain. This implies $\psi \in F$ (defined in (2.23) $)$ and therefore the contradiction $\psi \notin G$.

Now assume $\psi \in G$ and $\left\{v \in \psi_{\infty}: R(v)>0\right\}=\emptyset$. By our construction algorithm this is only possible if there exists a sequence of points $u_{i}=\left(x_{i}, t_{i}, K_{i}\right) \in \psi_{\infty}, i \in \mathbb{N}_{0}$, such that $t_{i}<d\left(u_{i}, u_{i+1}\right) \leq t_{i+1}$ and $t_{i} \geq t_{i+1}$ for $i \in \mathbb{N}_{0}$. This leads to $\psi \in F$ and again to $\psi \in G^{c}$.

We show $\mathbb{P}\left(\Psi \in F^{c}\right)=1$ and that the conditions (2.7), (2.21) and (2.22) have full measure, that is $\mathbb{P}(\Psi \in G)=1$. We start by calculating $\mathbb{P}\left(\Psi \in F_{n}(s, t, B)\right)$ for $n \in \mathbb{N}$, $s<t$ and $B \subset \mathbb{R}^{d}$. Positive constants (depending only on the dimension) occuring in this calculation are denoted by $c_{i}, i \geq 1$. Using assumption (2.2) and recalling the definition $B^{\prime}=B \times \mathbb{R}_{+} \times \mathcal{K}^{d}$ we obtain

$$
\begin{aligned}
& \mathbb{P}\left(\Psi \in F_{n}(s, t, B)\right) \\
& \quad \leq c_{1}^{n+1} \int \mathbf{1}\left\{u_{0} \in B^{\prime}\right\} \mathbf{1}\left\{s<d\left(u_{0}, u_{1}\right), \ldots, d\left(u_{n-1}, u_{n}\right) \leq t\right\}\left(\lambda^{d} \otimes \mathbb{Q}\right)^{n+1}\left(d\left(u_{0}, \ldots, u_{n}\right)\right) .
\end{aligned}
$$

With $f\left(u_{0}, \ldots, u_{n-1}\right):=\mathbf{1}\left\{u_{0} \in B^{\prime}\right\} \mathbf{1}\left\{s<d\left(u_{0}, u_{1}\right), \ldots, d\left(u_{n-2}, u_{n-1}\right) \leq t\right\}$ we conclude

$$
\begin{aligned}
& \mathbb{P}\left(\Psi \in F_{n}(s, t, B)\right) \\
& \leq c_{1}^{n+1} \int f\left(u_{0}, \ldots, u_{n-1}\right) \mathbf{1}\left\{s<d\left(u_{n-1}, u_{n}\right) \leq t\right\}\left(\lambda^{d} \otimes \mathbb{Q}\right)^{n+1}\left(d\left(u_{0}, \ldots, u_{n}\right)\right) \\
& \leq c_{1}^{n+1} \int f\left(u_{0}, \ldots, u_{n-1}\right) \mathbf{1}\left\{s<d\left(u_{n-1}, u_{n}\right) \leq t, t_{n-1} \leq t_{n}\right\}\left(\lambda^{d} \otimes \mathbb{Q}\right)^{n+1}\left(d\left(u_{0}, \ldots, u_{n}\right)\right) \\
& \quad+c_{1}^{n+1} \int f\left(u_{0}, \ldots, u_{n-1}\right) \mathbf{1}\left\{s<d\left(u_{n-1}, u_{n}\right) \leq t, t_{n-1} \geq t_{n}\right\}\left(\lambda^{d} \otimes \mathbb{Q}\right)^{n+1}\left(d\left(u_{0}, \ldots, u_{n}\right)\right) .
\end{aligned}
$$

We treat the summands separately, omitting the constant $c_{1}^{n+1}$ and using the definitions $\mu_{n}:=\left(\lambda^{d} \otimes \mathbb{Q}\right)^{n}, f_{n}:=f\left(u_{0}, \ldots, u_{n-1}\right)$ and $\left(x_{i}, t_{i}, K_{i}\right):=u_{i}, i=0, \ldots, n$. By (2.5) the first integral in (2.25) is bounded by

$$
\begin{aligned}
& \int f_{n} \mathbf{1}\left\{s<t_{n-1}+a\left(x_{n-1}, K_{n-1}, x_{n}, 0\right) \leq t\right\} \mu_{n+1}\left(d\left(u_{0}, \ldots, u_{n}\right)\right) \\
& +\int f_{n} \mathbf{1}\left\{s<t_{n}+a\left(x_{n-1}+\left(t_{n}-t_{n-1}\right) K_{n-1}, K_{n-1}, x_{n}, K_{n}\right) \leq t\right\} \mu_{n+1}\left(d\left(u_{0}, \ldots, u_{n}\right)\right) .
\end{aligned}
$$


We recall that $V_{d}(A)$ is the volume of $A \subset \mathbb{R}^{d}$. The integral (2.26) equals

$$
\begin{aligned}
& \int f_{n} \mathbf{1}\left\{t_{n-1} \leq t,\left(s-t_{n+1}\right)_{+}<a\left(x_{n-1}, K_{n-1}, x_{n}, 0\right) \leq t-t_{n-1}\right\} \mu_{n+1}\left(d\left(u_{0}, \ldots, u_{n}\right)\right) \\
& =\int f_{n} \mathbf{1}\left\{t_{n-1} \leq t, x_{n} \in x_{n-1}+\left[\left(t-t_{n-1}\right) K_{n-1} \backslash\left(s-t_{n-1}\right)_{+} K_{n-1}\right]\right\} \mu_{n+1}\left(d\left(u_{0}, \ldots, u_{n}\right)\right) \\
& \leq\left(t^{d}-s^{d}\right) \iint f_{n} V_{d}\left(K_{n-1}\right) \mu_{n}\left(d\left(u_{0}, \ldots, u_{n-1}\right)\right) \mathbb{Q}\left(d\left(t_{n}, K_{n}\right)\right),
\end{aligned}
$$

where we have used the inequalities $(t-c)^{d}-(s-c)^{d} \leq t^{d}-s^{d}$ and $(t-s)^{d} \leq t^{d}-s^{d}$ for $0 \leq c \leq s \leq t$. We define

$$
h(s, t):=\max \left\{t^{i}-s^{i}: i=1, \ldots, d\right\}, \quad 0 \leq s \leq t,
$$

and recall that $\rho(K)$ is the radius of the smallest all circumscribing $K \in \mathcal{K}^{d}$. The integral (2.27) equals

$$
\begin{aligned}
& \int f_{n} \mathbf{1}\left\{t_{n-1} \leq t,\left(s-t_{n}\right)_{+}<a\left(x_{n-1}+\left(t_{n}-t_{n-1}\right) K_{n-1}, K_{n-1}, x_{n}, K_{n}\right) \leq t-t_{n}\right\} \mu_{n+1}\left(d\left(u_{0}, \ldots, u_{n}\right)\right) \\
&= \int f_{n} \mathbf{1}\left\{t_{n-1} \leq t, x_{n} \in x_{n-1}+\left(t_{n}-t_{n-1}\right) K_{n-1}+\left(t-t_{n}\right)\left(K_{n-1}-K_{n}\right),\right. \\
&\left.\left.x_{n} \notin x_{n-1}+\left(t_{n}-t_{n-1}\right) K_{n-1}+\left(s-t_{n}\right)_{+}\left(K_{n-1}-K_{n}\right)\right\} \mu_{n+1}\left(d\left(u_{0}, \ldots, u_{n}\right)\right)\right\} \\
&=\iint \mathbf{1}\left\{t_{n-1} \leq t\right\} f_{n}\left[V_{d}\left(\left(t_{n}-t_{n-1}\right) K_{n-1}+\left(t-t_{n}\right)\left(K_{n-1}-K_{n}\right)\right)\right. \\
&\left.-V_{d}\left(\left(t_{n}-t_{n-1}\right) K_{n-1}+\left(s-t_{n}\right)_{+}\left(K_{n-1}-K_{n}\right)\right)\right] \mu_{n}\left(d\left(u_{0}, \ldots, u_{n-1}\right)\right) \mathbb{Q}\left(d\left(t_{n}, K_{n}\right)\right) \\
& \leq \iint f_{n} h(s, t) \sum_{i=0}^{d-1} \kappa_{d}\left(\begin{array}{c}
d \\
i
\end{array}\right)\left(t_{n}-t_{n-1}\right)^{i} \rho\left(K_{n-1}\right)^{i}\left(\rho\left(K_{n-1}\right)+\rho\left(K_{n}\right)\right)^{d-i} \\
& \mu_{n}\left(d\left(u_{0}, \ldots, u_{n-1}\right)\right) \mathbb{Q}\left(d\left(t_{n}, K_{n}\right)\right),
\end{aligned}
$$

where we have used Lemma 2.7 to obtain the inequality. Now we calculate an upper bound for the second summand in (2.25). This can be done as in (2.28) and (2.30) with the roles of $\left(t_{n}, K_{n}\right)$ and $\left(t_{n-1}, K_{n-1}\right)$ interchanged in the integrand. So we obtain

$$
\begin{gathered}
\int f\left(u_{0}, \ldots, u_{n-1}\right) \mathbf{1}\left\{s<d\left(u_{n-1}, u_{n}\right) \leq t, t_{n-1} \geq t_{n}\right\} \mu_{n+1}\left(d\left(u_{0}, \ldots, u_{n}\right)\right) \\
\leq 2\left(t^{d}-s^{d}\right) \iint f_{n} V_{d}\left(K_{n}\right) \mu_{n}\left(d\left(u_{0}, \ldots, u_{n-1}\right)\right) \mathbb{Q}\left(d\left(t_{n}, K_{n}\right)\right) \\
+2 \iint f_{n} h(s, t) \sum_{i=0}^{d-1} \kappa_{d}\left(\begin{array}{c}
d \\
i
\end{array}\right)\left(t_{n-1}-t_{n}\right)^{i} \rho\left(K_{n}\right)^{i}\left(\rho\left(K_{n-1}\right)+\rho\left(K_{n-1}\right)\right)^{d-i} \\
\mu_{n}\left(d\left(u_{0}, \ldots, u_{n-1}\right)\right) \mathbb{Q}\left(d\left(t_{n}, K_{n}\right)\right) .
\end{gathered}
$$

Combining (2.28)-(2.31) with (2.24) yields

$$
\begin{aligned}
& \mathbb{P}\left(\Psi \in F_{n}(s, t, B)\right) \leq c_{1}^{n+1} h(s, t) c_{2} \iint f_{n}\left(\left(t_{n-1} \vee 1\right)+\left(t_{n} \vee 1\right)\right)^{d} \\
&\left(\left(\rho\left(K_{n-1}\right) \vee 1\right)+\left(\rho\left(K_{n}\right) \vee 1\right)\right)^{d} \mu_{n}\left(d\left(u_{0}, \ldots, u_{n-1}\right)\right) \mathbb{Q}\left(d\left(t_{n}, K_{n}\right)\right) .
\end{aligned}
$$


Since the integrand in (2.24) equals $f_{n+1}$, we obtain recursively

$$
\begin{aligned}
& \mathbb{P}\left(\Psi \in F_{n}(s, t, B)\right) \leq V_{d}(B) c_{3}^{n} c_{4} h(s, t)^{n} \\
& \int \prod_{i=1}^{n}\left(\left(t_{i-1} \vee 1\right)+\left(t_{i} \vee 1\right)\right)^{d}\left(\left(\rho\left(K_{i-1}\right) \vee 1\right)+\left(\rho\left(K_{i}\right) \vee 1\right)\right)^{d} \mathbb{Q}^{n+1}\left(d\left(\left(t_{0}, K_{0}\right), \ldots,\left(t_{n}, K_{n}\right)\right)\right) .
\end{aligned}
$$

It is convenient to introduce a random vector $\left(\left(\tau_{0}^{\prime}, Y_{0}\right), \ldots,\left(\tau_{n}^{\prime}, Y_{n}\right)\right)$ with distribution $\mathbb{Q}^{n+1}$ and to define $\tau_{i}:=\tau_{i}^{\prime} \vee 1$ and $\rho_{i}:=\rho\left(Y_{i}\right) \vee 1$ for $i=0, \ldots, n$. Then inequality (2.32) can be written as

$$
\mathbb{P}\left(\Psi \in F_{n}(s, t, B)\right) \leq V_{d}(B) c_{3}^{n} c_{4} h(s, t)^{n} \mathbb{E}\left[\left(\prod_{i=1}^{n}\left(\tau_{i-1}+\tau_{i}\right)\right)^{d}\left(\prod_{i=1}^{n}\left(\rho_{i-1}+\rho_{i}\right)\right)^{d}\right] .
$$

Now we can use the elementary inequality

$$
\prod_{i=1}^{n}\left(a_{i-1}+a_{i}\right) \leq 2^{n}\left(\prod_{i=1}^{n} a_{i-1} a_{i}\right) \leq 2^{n} \prod_{i=0}^{n} a_{i}^{2}
$$

that holds whenever $a_{0}, \ldots, a_{n} \geq 1$. This gives

$$
\begin{aligned}
\mathbb{P}\left(\Psi \in F_{n}(s, t, B)\right) & \leq V_{d}(B) c_{3}^{n} c_{4} h(s, t)^{n} 2^{2 n d} \mathbb{E}\left[\prod_{i=0}^{n} \tau_{i}^{2 d} \rho_{i}^{2 d}\right] \\
& =V_{d}(B) c_{3}^{n} c_{4} h(s, t)^{n}\left(4^{d}\right)^{n}\left(\mathbb{E}\left[\tau_{0}^{2 d} \rho_{0}^{2 d}\right]\right)^{n+1} .
\end{aligned}
$$

Assumption (2.3) implies that

$$
\mathbb{P}\left(\Psi \in F_{n}(s, t, B)\right) \leq V_{d}(B) c_{5} c_{6}^{n} h(s, t)^{n} .
$$

Now we define $b_{i}:=\left(i /\left(c_{6}+1\right)\right)^{1 / d}, i \in \mathbb{N}_{0}$. By definition (2.29) of $h$

$$
h\left(b_{i-1}, b_{i}\right) \leq \frac{1}{c_{6}+1} .
$$

Hence $\lim _{n \rightarrow \infty} \mathbb{P}\left(\Psi \in F_{n}\left(b_{i-1}, b_{i}, W_{j}\right)\right)=0, i, j \in \mathbb{N}$, so that (2.20) implies $\mathbb{P}(\Psi \in F)=0$.

We now show that (2.7), (2.21) and (2.22) have full measure. The probability that $\Psi$ does not satisfy (2.7) can be bounded by

$$
\mathbb{E} \sum_{\left(u_{0}, u_{1}, u_{2}\right) \in \Psi^{(3)}} \mathbf{1}\left\{d\left(u_{0}, u_{1}\right)=d\left(u_{0}, u_{2}\right)\right\}+\mathbb{E} \sum_{\left(u_{0}, u_{1}, u_{2}, u_{2}\right) \in \Psi^{(4)}} \mathbf{1}\left\{d\left(u_{0}, u_{1}\right)=d\left(u_{2}, u_{3}\right)\right\},
$$

where the sum is taken over all triples resp. quadruples of points of $\Psi$ with pairwise different entries. We recall the definition (2.1) of the factorial moment measure $\nu^{(k)}$ for $k \in \mathbb{N}$ and that (2.2) holds for $\Psi$. Together with the definition (2.5) of $d$ it follows that the first summand of (2.33) is bounded by

$$
a \iiint \mathbf{1}\left\{x_{2} \in \partial A\left(u_{0}, u_{1},\left(s_{2}, K_{2}\right)\right)\right\} d x_{2} \mathbb{Q}\left(d\left(s_{2}, K_{2}\right)\right) \mu_{2}\left(d\left(u_{0}, u_{1}\right)\right),
$$


where $A\left(u_{0}, u_{1},\left(s_{2}, K_{2}\right)\right)$ is a convex body, depending on $u_{0}, u_{1}$ and $\left(s_{2}, K_{2}\right)$ but not on $x_{2}$. Hence this integral is zero. The second summand in (2.33) can be treated analogously. Thus we obtain that $\Psi$ satisfies (2.7) almost surely. That $\Psi$ does also satisfy (2.22) almost surely can be shown with the same type of argument.

Finally we prove that $\mathbb{P}(\operatorname{card}\{v \in \Psi \backslash\{u\}: d(u, v) \leq r\}<\infty$ for all $u \in \Psi)=1$ for all $r>0$. It is sufficient to show, that

$$
\mathbb{E} \sum_{(u, v) \in \Psi^{(2)}} \mathbf{1}\{\operatorname{card}\{v \in \Psi \backslash\{u\}: d(u, v) \leq r\}<\infty\} \mathbf{1}\left\{u \in W_{j} \times \mathbb{R}_{+} \times \mathcal{K}^{d}\right\}
$$

is finite for all $j \in \mathbb{N}$ and $r>0$. For fixed $j$ and $r$ this expectation is bounded by

$$
a^{2} \iiint \mathbf{1}\left\{x \in W_{j}, d((x, s, K),(y, t, L) \leq r\} d x d y \mathbb{Q}^{2}(d((s, K),(t, L))),\right.
$$

which in turn is bounded by

$$
\begin{aligned}
& a^{2} \iiint \mathbf{1}\left\{x \in W_{j}, s \leq t, s+a(x, K, y, 0) \leq r\right\} d x d y \mathbb{Q}^{2}(d((s, K),(t, L))) \\
& +a^{2} \iiint \mathbf{1}\left\{x \in W_{j}, s \leq t, t+a(x+(t-s) K, K, y, L) \leq r\right\} d x d y \mathbb{Q}^{2}(d((s, K),(t, L))) \\
& +a^{2} \iiint \mathbf{1}\left\{x \in W_{j}, t \leq s, t+a(y, L, x, 0) \leq r\right\} d x d y \mathbb{Q}^{2}(d((s, K),(t, L))) \\
& +a^{2} \iiint \mathbf{1}\left\{x \in W_{j}, t \leq s, s+a(y+(s-t) L, L, x, K) \leq r\right\} d x d y \mathbb{Q}^{2}(d((s, K),(t, L))) .
\end{aligned}
$$

The first two summands equal

$$
\begin{aligned}
& a^{2} \iiint \mathbf{1}\left\{x \in W_{j}, s \leq t, s \leq r\right\} \mathbf{1}\{y \in x+(r-s) K\} d x d y \mathbb{Q}^{2}(d((s, K),(t, L))) \\
& +a^{2} \iiint \mathbf{1}\left\{x \in W_{j}, s \leq t, t \leq r\right\} \mathbf{1}\{y \in x+(t-s) K+(r-t)(K-L)\} \\
& d x d y \mathbb{Q}^{2}(d((s, K),(t, L))) .
\end{aligned}
$$

This sum is bounded by

$$
\begin{gathered}
a^{2} \iiint \mathbf{1}\left\{x \in W_{j}, s \leq t, s \leq r\right\} \kappa_{d}(r-s)^{d} \rho(K)^{d} d x d y \mathbb{Q}^{2}(d((s, K),(t, L))) \\
+a^{2} \iiint \mathbf{1}\left\{x \in W_{j}, s \leq t, t \leq r\right\} \kappa_{d}[(t-s) \rho(K)+(r-t)(\rho(K)+\rho(L))]^{d} \\
d x d y \mathbb{Q}^{2}(d((s, K),(t, L))) .
\end{gathered}
$$

By (2.3) both summands are finite. By symmetry the third and fourth summand in (2.35) can be treated in the same way. Therefore (2.34) is finite for all $j \in \mathbb{N}$ and $r>0$. 


\section{Absence of percolation}

In this section we consider a point process $\Psi=\left\{\left(X_{n}, T_{n}, Z_{n}\right): n \geq 1\right\}$ satisfying the moment assumptions (2.2), where the probability measure $\mathbb{Q}$ is assumed to satisfy (2.3) and, moreover, to be concentrated on the set of all strictly convex bodies. In addition we assume $\Psi$ is non-empty and stationary, that is $\mathbb{P}(x+\Psi \in \cdot)$ does not depend on $x \in \mathbb{R}^{d}$. The intensity of $\Psi$ is defined by $\gamma_{\Psi}:=\mathbb{E} \Psi\left([0,1]^{d} \times \mathbb{R}_{+} \times \mathcal{K}^{d}\right)$. By (2.2) this is a finite number, while $\Psi \neq \emptyset$ implies $\gamma_{\Psi}>0$.

Due to Theorem 2.1 there exists a $\mathbb{P}$-a.s. unique growth-maximal hard-core model $\Psi^{*}=\left\{\left(X_{n}, T_{n}, Z_{n}, R_{n}\right): n \geq 1\right\}$ based on $\Psi$. We define

$$
Z:=Z(\Psi):=\bigcup_{n=1}^{\infty} X_{n}+R_{n} Z_{n}=\bigcup_{n: R_{n}>0} X_{n}+R_{n} Z_{n}
$$

that is the union of all grains (which started growing). Note that $X_{n} \in Z$ if $R_{n}=0$. We say that $Z$ (or the growth-maximal hard-core model) percolates, if $Z$ contains an unbounded connected component. To be more exact we introduce a graph with vertex set $\Psi_{+}:=\left\{\left(X_{n}, T_{n}, Z_{n}\right): R_{n}>0\right\}$. Two different points $\left(X_{m}, T_{m}, Z_{m}\right),\left(X_{n}, T_{n}, Z_{n}\right) \in \Psi_{+}$ share an edge if they are neighbours, that is if $\left(X_{m}+R_{m} Z_{m}\right) \cap\left(X_{n}+R_{n} Z_{n}\right) \neq \emptyset$. A cluster is a connected component of this graph.

As $\mathbb{P}(\Psi \neq \emptyset)=1$, the second defining property (ii) of a growth-maximal hard-core model given in the introduction together with Theorem 2.1 implies that almost surely $\Psi_{+}$ is non-empty too. Since $\Psi_{+}$is stationary (by the translation invariance of $R$ ) we have in fact that $\mathbb{P}\left(\operatorname{card} \Psi_{+}=\infty\right)=1$. Our aim in this section is to verify the following theorem.

Theorem 3.1. Almost surely there are no infinite clusters.

This theorem implies in particular that the random set $Z$ does not percolate. Our proof is based on some ideas in [4] and [2]. We begin with the following lemma.

Lemma 3.2. Almost surely any point $\left(X_{n}, T_{n}, Z_{n}\right) \in \Psi_{+}$has exactly one earlier neighbour.

Proof: Assume without loss that $\Psi \in H$ with $H$ being the set from Proposition 2.3. In addition suppose $u=(x, s, K) \in \Psi$ with $R(u):=R(\Psi, u)>0$ where $R(\cdot, \cdot)$ is the function introduced in Proposition 2.3. By growth-maximality $u$ has at least one earlier neighbour. If $u$ has more than one earlier neighbour, then for two different neighbours $v=(y, t, L)$ and $w=(z, r, M)$ of $u$ one of the following cases must occur:

$$
\begin{aligned}
& R(u)=R(v)+t-s=R(w)+r-s>0 \\
& R(u)=R(v)+t-s>0, R(u)+s>R(w)+r>0 \\
& R(u)+s>R(v)+t>0, R(u)+t>R(w)+r>0 .
\end{aligned}
$$

We need to show that all three cases (3.2)-(3.4) have probability zero. In order to understand the argument we first illustrate each of the three cases under the additional assumption $t \leq s \leq r$. Because of the construction algorithm from the proof of Proposition 2.3 it follows from (3.2) that $R(u)=a(x+(t-s) K, K, y, L)=a(x, K, z+(r-s) M, M)+r-s$. In the case (3.3) we have $R(u)=a(x+(t-s) K, K, y, L)=a(x, K, z+R(w) M, 0)$. 
Moreover there exists an $n \in \mathbb{N}$ such that $R(w)$ can be replaced by an expression depending on $v$ and a neighbour $w_{1}=\left(z_{1}, r_{1}, M_{1}\right) \in \Psi_{n} \backslash\{u, v\}$, where $\Psi_{n}$ is defined in the construction algorithm in the proof of Proposition 2.3. For $s \geq r_{1}$ we have either $R(w)=a\left(x+\left(s-r_{1}\right) M, M, z_{1}, M_{1}\right)$ or $R(w)=a\left(z, M, z_{1}+R\left(w_{1}\right) M_{1}, 0\right)$. If $s<r_{1}$ we have either that $R(w)=a\left(x, M, z_{1}+\left(r_{1}-s\right) M_{1}, M_{1}\right)+\left(r_{1}-s\right)$ or that $R(w)=a\left(z, M, z_{1}+R\left(w_{1}\right) M_{1}, 0\right)$. Now $R\left(w_{1}\right)$ can be replaced in the same way using $w_{1}$ and a neighbour $w_{2} \in \Psi_{m} \backslash\left\{u, v, w_{1}\right\}, m \leq n$. After a finite number of steps this procedure ends and we have $a(x, K, z+R(w) M, 0)=a\left(x, K, z+f\left(w, w_{1}, \ldots, w_{k}\right) M, 0\right)$ for some $k \geq 0$. The third case leads to $R(u)=a(x, K, y+R(v) L, 0)=a(x, K, z+R(w) M, 0)$. We can apply the same replacing routine as in the second case for $R(v)$ and $R(w)$.

In the case (3.2) we have that

$$
\begin{aligned}
a\left(x+(t-s)_{+} K, K, y+(t-s)_{+} L, L\right) & \\
& =a\left(x+(r-s)_{+} K, K, z+(s-r)_{+} M, M\right)+(r-s)_{+}-(t-s)_{+}>0
\end{aligned}
$$

where $b_{+}:=\max \{b, 0\}$ for $b \in \mathbb{R}$. In the other two cases the preceding replacement process yields countable families $F_{n}, n \geq 1$, of functions $f:\left(\mathbb{R}^{d} \times \mathbb{R}_{+} \times \mathcal{K}^{d}\right)^{n} \rightarrow \mathbb{R}_{+}$ such that the following is true. There exist $k, l, m \geq 1, f_{1} \in F_{k}, f_{2} \in F_{l}, f_{3} \in F_{m}$ and $\left(v_{1}, \ldots, v_{k}\right) \in(\Psi \backslash\{u\})^{(k)},\left(w_{1} \ldots, w_{l}\right) \in(\Psi \backslash\{u\})^{(l)}$ as well as $\left(w_{1}^{\prime}, \ldots, w_{m}^{\prime}\right) \in(\Psi \backslash\{u\})^{(m)}$ such that

$$
a\left(x+(t-s)_{+} K, K, y+(t-s)_{+} L, L\right)+(t-s)_{+}=a\left(x, K, z+f_{1}\left(v_{1}, \ldots, v_{k}\right) M, 0\right)>0
$$

or

$$
a\left(x, K, y+f_{2}\left(w_{1}, \ldots, w_{l}\right) L, 0\right)=a\left(x, K, z+f_{3}\left(w_{1}^{\prime}, \ldots, w_{m}^{\prime}\right) M, 0\right)>0,
$$

Using that the set $F:=\cup_{n \in \mathbb{N}} F_{n}$ is countable and assumption (2.2) on the factorial moment measures it is not difficult to see that the probabilities of all three cases (3.5)(3.6) are zero, provided the set of $x \in \mathbb{R}^{d}$ satisfying (3.5)-(3.7) is of Lebesgue measure zero. In the case (3.7) this means that

$$
\int \mathbf{1}\left\{a\left(x, K, K^{\prime}, 0\right)=a\left(x, K, K^{\prime \prime}, 0\right)>0\right\} d x=0
$$

whenever $K$ is strictly convex and the interiors of $K^{\prime}$ and $K^{\prime \prime}$ do not intersect. In fact, if the previous properties are satisfied and $r:=a\left(x, K, K^{\prime}, 0\right)=a\left(x, K, K^{\prime \prime}, 0\right)>0$ for some $x \in \mathbb{R}^{d}$, then the infimum $r=\inf \left\{t>0:(x+t K) \cap\left(K^{\prime} \cup K^{\prime \prime}\right) \neq \emptyset\right\}$ is attained in two different points in the boundary of $K^{\prime} \cup K^{\prime \prime}$. It follows from the Lipschitz property of the $K$-distance and the strict convexity of $K$ that the set of points $x$ with this property has Lebesgue measure 0. The detailed argument (even for sets more general than $K^{\prime} \cup K^{\prime \prime}$ ) can be found in [6]. The cases (3.5) and (3.6) can be treated similarly.

Two different points $\left(X_{m}, T_{m}, Z_{m}\right),\left(X_{n}, T_{n}, Z_{n}\right) \in \Psi_{+}$form a doublet if $T_{m}+R_{m}=$ $T_{n}+R_{n}$. This means that the points stop each other mutually.

Lemma 3.3. Almost surely any cluster contains at most one doublet while any finite cluster contains exactly one doublet. 
ProOF: For $u=(x, s, K) \in \Psi$ we write $R(u):=R(\Psi, u)$ and $S(u):=s+R(u)$. Consider a cluster $\Xi \subset \Psi_{+}$and assume that $\{u, v\},\left\{u^{\prime}, v^{\prime}\right\}$ are two different doublets in $\Xi$. Lemma 3.2 implies that $\{u, v\} \cap\left\{u^{\prime}, v^{\prime}\right\}=\emptyset$. (In the following we ignore $\mathbb{P}$-null sets.) Assume (without loss) that $S(u) \geq S\left(u^{\prime}\right)$ and let $u_{0}, u_{1}, \ldots, u_{m}$ be a path in $\Psi_{+}$such that $u_{0}=u, u_{1} \notin\{u, v\}, u_{m}=u^{\prime}$, and $v^{\prime} \notin\left\{u_{1}, \ldots, u_{m}\right\}$. Lemma 3.2 implies that $S(u)<$ $S\left(u_{1}\right)$ (otherwise $u$ would have two earlier neighbours $v$ and $u_{1}$ ) and then, recursively, $S\left(u_{i}\right)<S\left(u_{i+1}\right)$ for all $i \in\{0, \ldots, m-1\}$. Therefore $S\left(u_{m-1}\right)<S\left(u_{m}\right)=S\left(u^{\prime}\right)=S\left(v^{\prime}\right)$, contradicting the fact that $u^{\prime}$ has only one earlier neighbour. Therefore the two doublets cannot be connected by a path, so that $\Xi$ can have at most one doublet. If $\Xi$ is finite, then any $u \in \Xi$ that minimizes the function $S$ on $\Xi$ must be a member of a doublet.

Proof of TheOrem 3.1; Assume that $\Xi \subset \Psi_{+}$is an infinite cluster without a doublet. Take $u_{1} \in \Xi$ and let $u_{2}$ be the (unique) earlier grain neighbour of $u_{1}$. By assumption we have $S\left(u_{1}\right)>S\left(u_{2}\right)$. Continuing this way we obtain an infinite sequence $u_{1}, u_{2}, \ldots$ of different points in $\Psi$ such that $R\left(u_{n}\right)>0$ and $S\left(u_{n}\right)>S\left(u_{n+1}\right)$ for all $n \geq 1$. Now assume that there are $s<t$ such that $s<S\left(u_{n}\right) \leq t$ for all $n \geq 1$. Then Lemma 2.2 implies that $s<d\left(u_{n}, u_{n+1}\right) \leq t$ for all $n \geq 1$. We have shown in the proof of Theorem 2.1 that his event has probability zero.

In view of Lemma 3.3 it remains to prove that there are no infinite clusters with exactly one doublet. It is here, where stationarity plays a crucial role. In contrast to [4, 2] we use the mass-transport principle (see e.g. [8])

$$
\mathbb{E} \iint 1\{u \in B\} g\left(\Psi_{+}, u, v\right) \Psi_{+}(d v) \Psi_{+}(d u)=\mathbb{E} \iint 1\{v \in B\} g\left(\Psi_{+}, u, v\right) \Psi_{+}(d u) \Psi_{+}(d v),
$$

that holds for all measurable $B \subset \mathbb{R}^{d} \times \mathbb{R}_{+} \times \mathcal{K}^{d}$ and all non-negative measurable functions $g$, provided that $g$ is translation-invariant. For $v \in \Psi_{+}$define $C(v) \equiv C\left(\Psi_{+}, v\right) \subset \Psi_{+}$as the cluster containing $v$. Let $\Psi_{\infty}$ be the set of all $v \in \Psi_{+}$such that $C(v)$ is infinite and has a doublet. Define $g\left(\Psi_{+}, u, v\right):=1$ if $u \in \Psi_{\infty}, v \in C(u)$, and if $u$ is the lexicographically smallest point of the doublet of $C(v)$. Otherwise, set $g\left(\Psi_{+}, u, v\right):=0$. With this choice of $g$ and $B:=[0,1]^{d} \times \mathbb{R}_{+} \times \mathcal{K}^{d}$ the right-hand side of (3.9) is at most $\mathbb{E} \Psi_{+}[0,1]^{d} \leq \gamma_{\Psi}$. The left-hand side vanishes if the intensity $\gamma_{\infty}$ of the stationary point process $\Psi_{\infty}$ is zero. Otherwise $\Psi_{\infty}$ has infinitely many points and the left-hand side is infinite. This shows that $\gamma_{\infty}=0$, as asserted.

\section{A central limit theorem}

In this section we assume that $\Psi$ is an independently marked Poisson process on $\mathbb{R}^{d}$ with intensity 1 and mark space $[0, \infty) \times \mathcal{K}^{d}$ satisfying $\mathbb{P}$-a.s. the restrictions $T_{0}=0$ and

$$
B^{d} \subset Z_{0} \subset c B^{d}
$$

for some fixed $c \geq 1$. The assumption $T_{0}=0$ means that all grains are born at the same time (taken as 0 without further restriction of generaliy), while (4.1) implies that all growth times are strictly positive and bounded from below and above independently 
of the grain shape. Using stabilization arguments $([9,10])$ we shall prove a central limit theorem for the growth times $R_{n}, n \in \mathbb{N}$.

Let $g$ be a finite kernel from $\mathbb{R}_{+} \times \mathbb{R}^{d} \times \mathcal{K}_{0}^{d}$ to $\mathbb{R}^{d}$, where $\mathcal{K}_{0}^{d}$ is the set of all $K \in \mathcal{K}^{d}$ satisfying $B^{d} \subset K \subset c B^{d}$. We assume $g$ to be translation invariant, that is $g(t, x, K, A)=$ $g(t, x+y, K, A+y)$ for all $y \in \mathbb{R}^{d}$. In addition we let $\mathbf{N}_{0}$ denote the set of all $\psi \in \mathbf{N}$, such that for all $(x, s, K) \in \psi$ we have $s=0$ and $K \in \mathcal{K}_{0}^{d}$. We use the abbreviation $(x, K):=(x, 0, K)$ for all $(x, 0, K) \in \psi$ and $\psi \in \mathbf{N}_{0}$ and suppress the birth time in all other expressions as well. For all $x, y \in \mathbb{R}^{d}$ and $K, L \in \mathcal{K}_{0}^{d}$ define $\psi^{(x, K)}:=\psi \cup\{(x, K)\}$ and $\psi^{(x, K),(y, L)}:=\psi \cup\{(x, K),(y, L)\}$ if such a union lies in $\mathbf{N}_{0}$ and $\psi^{(x, K)}:=\psi$ resp. $\psi^{(x, K),(y, L)}:=\psi$ otherwise. Set

$$
\rho(\psi, x, K, A):=g\left(R\left(\psi^{(x, K)}, x, K\right), x, K, A\right) \mathbf{1}_{\left\{\operatorname{card}\left(\psi^{(x, K)}\right)>1\right\}}
$$

for all $\psi \in \mathbf{N}_{0}, x \in \mathbb{R}^{d}, K \in \mathcal{K}_{0}^{d}$ and measurable $A \subset \mathbb{R}^{d}$, where $R$ is the function defined in Proposition 2.3. With

$$
\mu(\psi, A):=\sum_{(x, K) \in \psi} \rho(\psi, x, K, A)
$$

for $\psi \in \mathbf{N}_{0}$ and measurable $A \subset \mathbb{R}^{d}$ we define a measure $\mu(\psi)(\cdot):=\mu(\psi, \cdot)$. Define the observation windows $W_{1}:=[-1 / 2,1 / 2]^{d}$ and $W_{n}:=n^{1 / d} W_{1}$ for $n \geq 2$. Furthermore set $\psi_{A}:=\psi \cap\left(A \times \mathcal{K}_{0}^{d}\right)$.

Theorem 4.1. Assume there exist $\alpha, \beta>0$ such that the translation invariant kernel $g$ in (4.2) satisfies the growth bound

$$
g\left(t, x, K, \mathbb{R}^{d}\right) \leq \alpha t^{\beta}, \quad t \geq 0, x \in \mathbb{R}^{d}, K \in \mathcal{K}_{0}^{d} .
$$

Moreover suppose that $f: W_{1} \rightarrow \mathbb{R}$ is a bounded, almost everywhere continuous function. Then the limit $\sigma_{\mu, f}:=\lim _{n \rightarrow \infty} \operatorname{Var}\left(\int_{W_{1}} f d \mu_{n}^{\rho}\right) / n$ exists and as $n \rightarrow \infty$

$$
\frac{1}{\sqrt{n}}\left(\int_{W_{n}} f\left(n^{-1 / d} x\right) \mu\left(\Psi_{W_{n}}, d x\right)-\mathbb{E}\left[\int_{W_{n}} f\left(n^{-1 / d} x\right) \mu\left(\Psi_{W_{n}}, d x\right)\right]\right) \stackrel{d}{\rightarrow} \mathcal{N}\left(0, \sigma_{\mu, f}\right) .
$$

We will derive this theorem from Theorem 2.1 and 2.2 from [10] starting with showing that the growth times $R\left(\psi^{(y, L)}, y, L\right)$ stabilize. Here we borrow heavily from [9] several times.

For $\psi \in \mathbf{N}_{0}, y \in \mathbb{R}^{d}$ and $K \in \mathcal{K}_{0}^{d}$ define

$$
D(\psi, y, L):=\inf \{a(y, L, x, 0):(x, K) \in \psi \backslash\{(y, L)\}\},
$$

with $a$ as in (2.4). If $(y, L) \in \psi$ then $D(\psi, y, L)$ is an upper bound for the growth time $R(\psi, y, L)$ of the grain $(y, L)$.

We recall the function $d$ defined in (2.5) and the constant $c \geq 1$ from assumption (4.1). Below we will use that

$$
\frac{1}{2 c}\|x-y\| \leq d(x, K, y, L) \leq \frac{1}{2}\|x-y\|, \quad x, y \in \mathbb{R}^{d}, K, L \in \mathcal{K}_{0}^{d},
$$

provided that $B^{d} \subset K \subset c B^{d}$ and $B^{d} \subset L \subset c B^{d}$. 
By a (finite) descending chain in $\psi \in \mathbf{N}_{0}$ we mean a finite sequence $u_{0}, \ldots, u_{n}(n \geq 1)$ of distinct points of $\psi$ for which $d\left(u_{i-1}, u_{i}\right) \geq d\left(u_{i}, u_{i+1}\right)$ for all $i \in\{1, \ldots, n-1\}$. For $\psi \in \mathbf{N}_{0}, y \in \mathbb{R}^{d}$ and $L \in \mathcal{K}_{0}^{d}$ a triple $(x, K, r) \in \mathbb{R}^{d} \times \mathcal{K}_{0}^{d} \times(0, \infty)$ belongs to the set $A(\psi, y, L)$ if there exists a descending chain $u_{0}, \ldots, u_{n}$ in $\psi^{(y, L)}$, such that $u_{0}=(y, L)$, $u_{n}=(x, K), d\left(u_{0}, u_{1}\right) \leq D\left(\psi^{(y, L)}, y, L\right)$ and $r=d\left(u_{n-1}, u_{n}\right)$. We define

$$
S(\psi, y, L):=B\left(y, 2 c D\left(\psi^{(y, L)}, y, L\right)\right) \cup \bigcup_{(x, K, r) \in A(\psi, y, L)} B(x, 2 c r),
$$

if $\psi \backslash\{(y, L)\} \neq \emptyset$ and $S(\psi, y, L)=\mathbb{R}^{d}$ otherwise and $S^{*}(\psi, y, L):=S(\psi, y, L) \times \mathcal{K}_{0}^{d}$.

Lemma 4.2. Let $\psi, \varphi \in \mathbf{N}_{0}, y \in \mathbb{R}^{d}$ and $L \in \mathcal{K}_{0}^{d}$. If $\psi \cap S^{*}(\psi, y, L)=\varphi \cap S^{*}(\psi, y, L)$ then $S(\psi, y, L)=S(\varphi, y, L)$.

Proof: We assume $\psi \backslash\{(y, L)\} \neq \emptyset$ since otherwise the result is trivial. Since $B\left(\left(y, 2 c D\left(\psi^{(y, L)}, y, L\right)\right) \subset S(\psi, y, L)\right.$ we have $D\left(\psi^{(y, L)}, y, L\right)=D\left(\varphi^{(y, L)}, y, L\right)$.

Take $(x, K, r) \in A(\psi, y, L)$. Thus there exists a descending chain $u_{0}, \ldots, u_{n} \in \psi^{(y, L)}$ such that $u_{0}=(y, L), u_{n}=(x, K), d\left(u_{0}, u_{1}\right) \leq D\left(\psi^{(y, L)}, y, L\right)$ and $r=d\left(u_{n-1}, u_{n}\right)$. We have $u_{m} \in S^{*}\left(\psi^{(y, L)}, y, L\right)$ for $1 \leq m \leq n$. Therefore $u_{m} \in \varphi$. So $(x, K, r) \in A(\varphi, y, L)$ and hence $A(\psi, y, L) \subset A(\varphi, y, L)$.

Consider $(x, K, r) \in A(\varphi, y, L)$. So there exists a descending chain $u_{0}, \ldots, u_{n} \in \varphi^{(y, L)}$ with $u_{0}=(y, L), u_{n}=(x, K), d\left(u_{0}, u_{1}\right) \leq D\left(\varphi^{(y, L)}, y, L\right)$ and $r=d\left(u_{n-1}, u_{n}\right)$. We show $u_{m} \in \psi$ for $1 \leq m \leq n$ by induction on $m$. For $m=1$ we have $d\left(u_{0}, u_{1}\right) \leq D\left(\varphi^{(y, L)}, y, L\right)$. Using (4.7) with $(y, L)$ and $u_{1}=\left(x_{1}, K_{1}\right)$ we obtain $\left\|y-x_{1}\right\| \leq 2 c D\left(\varphi^{(y, L)}, y, L\right)$. Therefore $u_{1} \in \varphi \cap S^{*}(\psi, y, L)$ and hence $u_{1} \in \psi$. Now assume our assertion holds for $u_{m}, 1 \leq m \leq$ $k-1$. Applying (4.7) with $u_{m}=\left(x_{m}, K_{m}\right)$ and $u_{m+1}=\left(x_{m+1}, K_{m+1}\right)$ yields $\left\|x_{m}-x_{m+1}\right\| \leq$ $2 c d\left(u_{m}, u_{m+1}\right)$. So $u_{k} \in \varphi \cap S^{*}(\psi, y, L)$ and thus $u_{k} \in \psi$ so that $A(\varphi, y, L) \subset A(\psi, y, L)$. Together with the inclusion proved above we deduce $A(\psi, y, L)=A(\varphi, y, L)$ and the assertion follows.

We recall from the proof of Lemma 2.4 the definition of a measurable set $G \subset H$ such that $\mathbb{P}(\Psi \in G)=1$.

Lemma 4.3. Suppose $\psi \in \mathbf{N}_{0} \cap G$ and $(y, L) \in \mathbb{R}^{d} \times \mathcal{K}_{0}^{d}$. If $S(\psi, y, L)$ is bounded, then it satisfies

$$
R\left(\psi^{(y, L)}, y, L\right)=R\left(\psi^{(y, L)} \cap S^{*}(\psi, y, L) \cup \varphi, y, L\right),
$$

for all finite $\varphi \in \mathbf{N}_{0}$ with $\varphi \subset \mathbb{R}^{d} \backslash S(\psi, y, L) \times \mathcal{K}_{0}^{d}$.

Proof: Suppose $S(\psi, y, L)$ is bounded and $\psi \in \mathbf{N}_{0}$. Since $S\left(\psi^{(y, L)}, y, L\right)=S(\psi, y, L)$ we assume w.l.o.g. $(y, L) \in \psi$. Moreover because of Lemma 4.2 it suffices to prove the equality in the case $\psi \subset S^{*}(\psi, y, L)$. Hence we assume this too. We note that since $\varphi$ is finite the set $\psi^{\prime}:=\psi \cup \varphi$ is an element of $\mathbf{N}_{0} \cap G$ and define $R(x, K):=R(\psi, x, K)$ and $R^{\prime}(x, K):=R\left(\psi^{\prime}, x, K\right)$.

Suppose $R(y, L)>R^{\prime}(y, L)$ and that $\left(x_{1}, K_{1}\right)$ is an earlier neighbour of $(y, L)$ in $\psi^{\prime}$. We assume for now $\left(x_{1}, K_{1}\right) \in \psi$. Since $\left(x_{1}, K_{1}\right)$ and $(y, L)$ are neighbours we obtain $\left(y+R^{\prime}(y, L) L\right) \cap\left(x_{1}+R^{\prime}\left(x_{1}, K_{1}\right) K_{1}\right) \neq \emptyset$. Because of the hard-core property on $\psi$ and $R(y, L)>R^{\prime}(y, L)$ it follows $R\left(x_{1}, K_{1}\right)<R^{\prime}\left(x_{1}, K_{1}\right)$. Suppose $\left(x_{2}, K_{2}\right)$ is an earlier 
neighbour of $\left(x_{1}, K_{1}\right)$ in $\psi$. The hard-core property on $\psi^{\prime}$ yields $R\left(x_{2}, K_{2}\right)>R^{\prime}\left(x_{2}, K_{2}\right)$. Let $\left(x_{3}, K_{3}\right)$ be an earlier neighbour of $\left(x_{2}, K_{2}\right)$ in $\psi^{\prime}$ and assume $\left(x_{3}, K_{3}\right) \in \psi$. Continuing this procedure leads to a sequence of points satisfying

$$
R(y, L)>R^{\prime}(y, L) \geq R^{\prime}\left(x_{1}, K_{1}\right)>R\left(x_{1}, K_{1}\right) \geq R\left(x_{2}, K_{2}\right)>R^{\prime}\left(x_{2}, K_{2}\right)>R^{\prime}\left(x_{3}, K_{3}\right) \ldots,
$$

terminating at $\left(x_{n}, K_{n}\right)$ if $\left(x_{n}, K_{n}\right) \in \varphi$ (so $n$ must be odd). The strict inequalities ensure that the points of this (possibly) terminating sequence are all different. By Lemma 2.2 we get

$$
R\left(x_{i}, K_{i}\right) \geq d\left(\left(x_{i}, K_{i}\right),\left(x_{i+1}, K_{i+1}\right)\right) \geq R\left(x_{i+1}, K_{i+1}\right)
$$

for odd $i \in \mathbb{N}$ and the same inequalities if we replace $R$ by $R^{\prime}$ and $i \in \mathbb{N}$ is even. Furthermore $R^{\prime}(y, L) \geq d\left((y, L),\left(x_{1}, K_{1}\right)\right) \geq R^{\prime}\left(x_{1}, K_{1}\right)$ holds. Therefore (4.8) implies

$$
d\left((y, L),\left(x_{1}, K_{1}\right)\right) \geq d\left(\left(x_{1}, K_{1}\right),\left(x_{2}, K_{2}\right)\right) \geq d\left(\left(x_{2}, K_{2}\right),\left(x_{3}, K_{3}\right)\right) \geq \ldots
$$

Thus the sequence forms a descending chain with $d\left((y, L),\left(x_{1}, K_{1}\right)\right) \leq D\left(\psi^{(y, L)}, y, L\right)$. If the sequence terminates at some point $\left(x_{n}, K_{n}\right) \in \psi^{\prime}$ then $\left(x_{n}, K_{n}\right) \in S^{*}(\psi, y, L)$ contradicting $\varphi \cap S^{*}(\psi, y, L)=\emptyset$. If the sequence does not terminate then $S(\psi, y, L)$ cannot be bounded which is also a contradiction. Hence if $R(y, L)>R^{\prime}(y, L)$ the assertion is shown. The case $R^{\prime}(y, L)>R(y, L)$ is proven similarly, this time starting with an earlier neighbour $\left(x_{1}, K_{1}\right)$ of $(y, L)$ in $\psi$.

Let $B(y, r):=\left\{x \in \mathbb{R}^{d}:\|x-y\| \leq r\right\}$ be the ball around $y \in \mathbb{R}^{d}$ of radius $r>0$ and $B^{*}(y, r):=B(y, r) \times \mathcal{K}_{0}^{d}$. Furthermore define for $\psi \in \mathbf{N}_{0} \cap G, y \in \mathbb{R}^{d}$ and $K \in \mathcal{K}_{0}^{d}$

$$
U(\psi, y, L):=\inf \{r>0: S(\psi, y, L) \subset B(y, r)\}
$$

and $U(\psi, y, L):=\infty$ otherwise. Because of the Lemma 4.3 we have

$$
R\left(\psi^{(y, L)}, y, L\right)=R\left(\psi^{(y, L)} \cap B^{*}(y, U(\psi, y, L) \cup \varphi, y, L),\right.
$$

for all finite $\varphi \in \mathbf{N}_{0}$ with $\varphi \subset \mathbb{R}^{d} \backslash B\left(y, U(\psi, y, L) \times \mathcal{K}_{0}^{d}\right.$. Hence we call $U$ a radius of stabilization of $R$ at $(y, L)$ with respect to $\psi$. In [10] the radius of stabilization $T$ of $\rho$ with respect to $\psi$ and $K$ is defined as

$$
\begin{array}{r}
T(\psi, K):=\inf \left\{r \geq 0: \rho\left(\left[\psi \cap B^{*}(0, t) \cup \varphi\right], 0, K, \mathbb{R}^{d}\right)=\rho\left(\left[\psi \cap B^{*}(0, t)\right], 0, K, \mathbb{R}^{d}\right)\right. \\
\text { for all finite } \left.\varphi \in \mathbf{N}_{0} \text { with } \varphi \subset\left(\mathbb{R}^{d} \backslash B(0, t)\right) \times \mathcal{K}_{0}^{d}\right\} .
\end{array}
$$

By definition $\rho\left(\psi, 0, K, \mathbb{R}^{d}\right)$ depends on $\psi$ only via the radius $R\left(\psi^{(0, K)}, 0, K\right)$. Because of (4.11) it follows that $T(\psi, K)$ is bounded by $U(\psi, 0, K)$. Now we deal with the tail behaviour of $U\left(\Psi, 0, Z_{0}\right)$.

Lemma 4.4. Let $0<\gamma<1$. Then there exist constants $\alpha, \beta>0$ such that

$$
\mathbb{P}\left(U\left(\Psi, 0, Z_{0}\right)>t\right) \leq \alpha \exp \left(-\beta t^{\gamma}\right), \quad t \geq 0 .
$$

The same holds for $U\left(\Psi^{\left(x, Z_{1}\right)}, 0, Z_{0}\right)$ for all $x \in \mathbb{R}^{d}$, where $Z_{1}$ is an independent copy of $Z_{0}$ and also independent of $\Psi$. 
Proof: We start with the first assertion. W.l.o.g we assume $t \geq 1$. Define $U:=$ $U\left(\Psi, 0, Z_{0}\right)$ and $D:=D\left(\Psi, 0, Z_{0}\right)$. Let $0<\varepsilon<1 /(d+1)$. It holds

$$
\{U>t\} \subset\left\{D>\frac{t^{\varepsilon}}{4 c}\right\} \cup\left\{D \leq \frac{t^{\varepsilon}}{4 c}, U>t\right\},
$$

where $c>1$ is the constant from the shape condition (4.1). Define $E$ as the set of all $\psi \in \mathbf{N}_{0}$, which contain a descending chain $\left(0, K_{0}\right), u_{1}, \ldots, u_{n}$ such that

$$
d\left(\left(0, K_{0}\right), u_{1}\right) \leq D\left(\psi, 0, K_{0}\right) \leq t^{\varepsilon} /(4 c)
$$

and $n \geq t^{1-\varepsilon} / 2$. For $\psi \in \mathbf{N}_{0}$ and $K_{0} \in \mathcal{K}_{0}^{d}$ such that $\psi^{\left(0, K_{0}\right)} \in \mathbf{N}_{0}, U\left(\psi, 0, K_{0}\right)>t$ and (4.13) holds we have $\psi^{\left(0, K_{0}\right)} \in E$. To see this, assume on the contrary that every descending chain of $\psi^{\left(0, K_{0}\right)}$ which satisfies (4.13) would consist of $n$ points with $n<t^{1-\varepsilon} / 2$. Since

$$
S\left(\psi, 0, K_{0}\right) \subset B\left(0,2 c(n+1) D\left(\psi, 0, K_{0}\right)\right)
$$

this would imply

$$
U\left(\psi, 0, K_{0}\right) \leq 2 c\left(\frac{t^{1-\varepsilon}}{2}+1\right) D\left(\psi, 0, K_{0}\right)
$$

by the definition (4.10) of $U$. Since $D\left(\psi, 0, K_{0}\right) \leq t^{\varepsilon} /(4 c), t \geq 1$, and $0<\varepsilon<1 /(d+1)$ this would lead to the contradiction $U\left(\psi, 0, K_{0}\right) \leq(3 / 4) t$. So we deduce

$$
\mathbb{P}(U>t) \leq \mathbb{P}\left(D>\frac{t^{\varepsilon}}{4 c}\right)+\mathbb{P}\left(\Psi^{\left(0, Z_{0}\right)} \in E\right) .
$$

Define $k:=\left\lceil\left(c_{1}+1\right) t^{\varepsilon d} /(4 c)^{d}\right\rceil$ and $s_{i}:=\left(i /\left(c_{1}+1\right)\right)^{1 / d}, i \geq 0$, where $c_{1}:=V_{d}(B(0,2 c))$ and $\lceil\cdot\rceil$ is the ceiling function. Hence $s_{k} \geq t^{\varepsilon} /(4 c)$. Set $m:=\left\lceil t^{1-\varepsilon} /(2 k)\right\rceil$ and $n_{0}:=\left\lceil t^{1-\varepsilon} / 2\right\rceil$. Note that if $t \rightarrow \infty$ then $m \rightarrow \infty$ as well. W.l.o.g assume $t \geq 1$ so large that $m \geq 3$. For $\psi \in E$ take a descending chain $\left(0, K_{0}\right), u_{1}, \ldots, u_{n}$ with $n \geq t^{1-\varepsilon} / 2$ and thus $n \geq n_{0}$. Since $n \geq\left\lceil t^{1-\varepsilon} / 2\right\rceil$ there exists $j \in\{1, \ldots, k\}$ and $l \in\left\{0, \ldots, n_{0}-m\right\}$ such that at least $m$ consecutive of the $n$ distances $d\left(\left(0, K_{0}\right), u_{1}\right)$ and $d\left(u_{i-1}, u_{i}\right), 2 \leq i \leq n$, lie in the interval $I_{j}:=\left[s_{j-1}, s_{j}\right]$. In addition

$$
\left\|x_{i}\right\| \leq\left(\frac{t^{1-\varepsilon}}{2}+1\right) \frac{2 c t^{\varepsilon}}{4 c} \leq t, \quad 1 \leq i \leq n_{0},
$$

where $x_{i}$ is the projection of $u_{i}$ onto the first coordinate, that is $u_{i}=\left(x_{i}, K_{i}\right)$. Hence $E \subset \cup_{j=1}^{k} E_{j}$ where $\psi$ belongs to $E_{j}$ if it contains a descending chain $\left(x_{1}, K_{1}\right), \ldots,\left(x_{m}, K_{m}\right)$ with $\left\|x_{1}\right\| \leq t$ and $d\left(\left(x_{i-1}, K_{i-1}\right),\left(x_{i}, K_{i}\right)\right) \in I_{j}, i \in\{1, \ldots, m-1\}$. Hence

$$
\mathbb{P}\left(\Psi^{\left(0, Z_{0}\right)} \in E\right) \leq \sum_{j=1}^{k} \mathbb{P}\left(\Psi \in E_{j}\right) .
$$

Furthermore we get

$$
\begin{aligned}
\mathbb{P}\left(\Psi \in E_{j}\right) \leq \mathbb{E}\left[\sum_{\left(\left(x_{1}, K_{1}\right), \ldots,\left(x_{m}, K_{m}\right)\right) \in \Psi^{(m)}} \mathbf{1}\left\{\left\|x_{1}\right\| \leq t, d\left(\left(x_{i}, K_{i}\right),\left(x_{i+1}, K_{i+1}\right)\right) \in I_{j}\right\}\right] \\
=\int \cdots \int \mathbf{1}\left\{\left\|x_{1}\right\| \leq t, d\left(\left(x_{i}, K_{i}\right),\left(x_{i+1}, K_{i+1}\right)\right) \in I_{j}\right\} d x_{1} \ldots d x_{m} Q\left(d K_{1}\right) \ldots Q\left(d K_{m}\right) .
\end{aligned}
$$


A calculation similar to the one following (2.24) yields

$$
\mathbb{P}\left(\Psi \in E_{j}\right) \leq c_{0} c_{1}^{m-1} t^{d}\left(s_{j}^{d}-s_{j-1}^{d}\right)^{m-1}=c_{0} t^{d}\left(\frac{c_{1}}{c_{1}+1}\right)^{m-1}
$$

with a constant $c_{0}>0$. Hence there exist constants $c_{2}, c_{3}>0$ such that

$$
\mathbb{P}\left(\Psi \in E_{j}\right) \leq c_{3} t^{d} \exp \left(-c_{2} m\right), \quad j=1, \ldots, k .
$$

Since $t^{\varepsilon d} \geq 1$ and $c>1$ we get $k \leq\left(c_{1}+2\right) t^{\varepsilon d}$. Using (4.15) and (4.16) we obtain

$$
\mathbb{P}\left(\Psi^{\left(0, Z_{0}\right)} \in E\right) \leq\left(c_{2}+2\right) t^{\varepsilon d} c_{3} t^{d} \exp \left(-c_{2} m\right) \leq c_{4} t^{d+\varepsilon d} \exp \left(-c_{5} t^{1-\varepsilon-\varepsilon d}\right)
$$

with constants $c_{4}, c_{5}>0$. Note that $1-\varepsilon-\varepsilon d>0$. Now we give a bound for the first summand in (4.14). By definition (4.6) of $D=D\left(\Psi, 0, Z_{0}\right)$ we have

$$
\mathbb{P}\left(D>\frac{t^{\varepsilon}}{4 c}\right) \leq \mathbb{P}\left(\Psi\left(B\left(0, \frac{t^{\varepsilon} \zeta\left(Z_{0}\right)}{4 c}\right) \times \mathcal{K}_{0}^{\prime}\right)=0\right) \leq \mathbb{P}\left(\Psi\left(B\left(0, \frac{t^{\varepsilon}}{4 c}\right) \times \mathcal{K}_{0}^{\prime}\right)=0\right),
$$

where $\zeta(K), K \in \mathcal{K}^{d}$, is the largest $r>0$ such that $B(0, r) \subset K$. The second inequality is due to the first inclusion in (4.1). Since $\Psi$ is a Poisson process this yields

$$
\mathbb{P}\left(D>\frac{t^{\varepsilon}}{4 c}\right) \leq \exp \left(-c_{6} t^{\varepsilon d}\right),
$$

where $c_{6}>0$. With (4.14) we get from the preceding two inequalities

$$
\mathbb{P}(U>t) \leq \exp \left(-c_{6} t^{\varepsilon d}\right)+c_{4} t^{d+\varepsilon d} \exp \left(-c_{5} t^{1-\varepsilon-\varepsilon d}\right),
$$

for all sufficiently large $t$. This implies the first assertion.

The second assertion can be shown using the same argumentation as above. But to be able to deal with the inserted point $\left(x, Z_{0}\right)$ we have to divide the event $E_{j}$ for $j=1, \ldots, k$ into events $E_{j, i}, i=1, \ldots, m$, where $E_{j, i}$ is the event that $E_{j}$ occurs and the $i$-th point of the descending chain of the configuration is $(x, K)$ for a fixed $K \in \mathcal{K}_{0}^{d}$. Hence the analogue of (4.15) consists of a double sum on the right-hand side. Since the arguments used to bound the probability of $E_{j}$ also hold for $E_{j, i}$ and because of the definition of $m$ we still obtain an sub-exponential decaying tail. We omit the details.

We define

$$
\begin{aligned}
& \rho_{n}(\psi, x, K, A):=\rho\left(n^{1 / d} \psi, n^{1 / d} x, K, n^{1 / d} A\right) \mathbf{1}_{W_{1}}(x), \\
& \tau(s):=\sup _{n \geq 1, x \in W_{1}} \mathbb{P}\left(T\left(n^{1 / d}\left(\left(n^{-1 / d} \Psi\right)_{W_{1}}-x\right), x, Z_{0}\right)>s\right), \quad s>0, \\
& \mu_{n}^{\rho}(A):=\sum_{(x, K) \in\left(n^{-1 / d} \Psi\right)_{W_{1}}} \rho_{n}\left(\left(n^{-1 / d} \psi\right)_{W_{1}}, x, K, A\right)
\end{aligned}
$$

for $\psi \in \mathbf{N}_{0}, x \in \mathbb{R}^{d}, K \in \mathcal{K}_{0}^{d}$ and $A \subset \mathbb{R}^{d}$. Note that

$$
\int_{W_{n}} f\left(n^{-1 / d} x\right) \mu\left(\Psi_{W_{n}}, d x\right) \stackrel{d}{=} \int_{W_{1}} f d \mu_{n}^{\rho} .
$$

This equation links (4.5) with the form in which the central limit theorem is formulated in Theorem 2.2 in [10]. The next lemma shows that (2.7), (2.8) and (2.10) in [10] are satisfied. 
Lemma 4.5. Suppose $Z_{1}$ is an independent copy of $Z_{0}$ and also independent of $\Psi$. Then

$$
\mathbb{P}\left(U\left(\Psi, 0, Z_{0}\right)<\infty\right)=\mathbb{P}\left(U\left(\Psi^{\left(x, Z_{1}\right)}, 0, Z_{0}\right)<\infty\right)=1 .
$$

Furthermore for $p>0$

$$
\begin{aligned}
& \sup _{n \geq 1, x \in W_{1}} \mathbb{E}\left[\rho_{n}\left(\left(n^{-1 / d} \Psi\right)_{W_{1}}, x, Z_{0}, \mathbb{R}^{d}\right)^{p}\right]<\infty, \\
& \sup _{n \geq 1, x, y \in W_{1}} \mathbb{E}\left[\rho_{n}\left(\left[\left(n^{-1 / d} \Psi\right)_{W_{1}}\right]^{\left(y, Z_{1}\right)}, x, Z_{0}, \mathbb{R}^{d}\right)^{p}\right]<\infty,
\end{aligned}
$$

and $\sup _{s \geq 1} s^{q} \tau(s)<\infty$ for some $q>d(150+6 / p)$.

Proof: The first assertion (4.19) follows directly from Lemma 4.4. For $p>0$ we have

$$
\begin{aligned}
\sup _{n \geq 1, y \in W_{1}} \mathbb{E}\left[\rho_{n}\left(\left(n^{-1 / d} \Psi\right)_{W_{1}}, y, Z_{0}, \mathbb{R}^{d}\right)^{p}\right] & =\sup _{n \geq 1, y \in W_{1}} \mathbb{E}\left[\rho\left(n^{1 / d}\left(n^{-1 / d} \Psi\right)_{W_{1}}, n^{1 / d} y, Z_{0}, \mathbb{R}^{d}\right)^{p}\right] \\
& =\sup _{n \geq 1, x \in W_{n}} \mathbb{E}\left[\rho\left(\Psi_{W_{n}}, x, Z_{0}, \mathbb{R}^{d}\right)^{p}\right] .
\end{aligned}
$$

The growth bound (4.4) and the definition of $\rho$ imply for all $n \geq 1$ and $x \in W_{n}$ that

$$
\mathbb{E}\left[\rho\left(\Psi_{W_{n}}, x, Z_{0}, W_{n}\right)^{p}\right] \leq \alpha^{p} \mathbb{E}\left[R\left(\left(\Psi_{W_{n}}\right)^{\left(x, Z_{0}\right)}, x, Z_{0}\right)^{\beta p} \mathbf{1}_{\left\{\operatorname{card}\left(\left(\Psi_{W_{n}}\right)^{\left(x, Z_{0}\right)}\right)>1\right\}}\right] .
$$

Define the random variable $Y_{n, x}:=R\left(\left(\Psi_{W_{n}}\right)^{\left(x, Z_{0}\right)}, x, Z_{0}\right) \mathbf{1}_{\left\{\operatorname{card}\left(\Psi_{W_{n}}\right)>0\right\}}$. For $t \leq \operatorname{diam}\left(W_{n}\right)$ we conclude from $(2.10)$ in 9 ]

$$
\mathbb{P}\left(Y_{n, x}>t\right) \leq \mathbb{P}\left(R\left(\left(\Psi_{W_{n}}\right)^{\left(x, Z_{0}\right)}, x, Z_{0}\right)>t\right) \leq \exp \left(-c_{0} t^{d}\right),
$$

where $c_{0}$ only depends on $W_{1}$. Trivially this inequality holds for $t>\operatorname{diam}\left(W_{n}\right)$ as well. This yields (4.20). Assertion (4.21) can be shown analogously, if we replace $Y_{n, x}$ by

$$
Y_{n, x, y}:=R\left(\left(\Psi_{W_{n}}\right)^{\left(x, Z_{0}\right),\left(y, Z_{1}\right)}, x, Z_{0}\right) .
$$

To prove the statement about $\tau$ we note

$$
\begin{aligned}
\tau(s) & =\sup _{n \geq 1, y \in W_{1}} \mathbb{P}\left(T\left(n^{1 / d}\left(n^{-1 / d} \Psi\right)_{W_{1}}-n^{1 / d} y, Z_{0}\right)>s\right) \\
& =\sup _{n \geq 1, x \in W_{n}} \mathbb{P}\left(T\left(\Psi_{W_{n}}-x, Z_{0}\right)>s\right) \\
& \leq \sup _{n \geq 1, x \in W_{n}} \mathbb{P}\left(U\left(\Psi_{W_{n}}-x, 0, Z_{0}\right)>s\right),
\end{aligned}
$$

where we use $T(\varphi, K) \leq U(\varphi, 0, K)$ for $\varphi \in \mathbf{N}_{0}, K \in \mathcal{K}_{0}^{d}$.

With some minor technical modifications we can use the proof of Lemma 4.4 to show that $\mathbb{P}\left(U\left(\Psi_{W_{n}}-x, 0, Z_{0}\right)>s\right)$ is sub-exponentially decaying independent of $n \geq 1$ and $x \in W_{n}$. The dependence on $n$ vanishes because it is possible to refer to $\Psi$ instead of $\Psi_{W_{n}}$ for all $n \in \mathbb{N}$ if necessary. The dependence on $x$ vanishes because of the translation invariance of the Lebesgue measure. We omit the details.

Proof of Theorem 4.1: Because of Lemma 4.5 the assumptions of Theorem 2.1 and 2.2 in [10] are satisfied. Due to (4.18) Theorem 4.1] is implied by these two theorems. 
Remark 4.6. The first inclusion in the assumption (4.1) has been used to achieve the bound in (4.17). As the proof shows, this bound can be replaced by the slightly weaker assumption

$$
\mathbb{P}\left(t B^{d} \subset Z_{0}\right) \geq 1-\exp \left(-(1 / t)^{\varepsilon}\right)
$$

for all sufficiently small $t>0$.

Consider a measurable function $h: \mathbb{R}_{+} \times \mathcal{K}_{0}^{d} \rightarrow \mathbb{R}_{+}$. If we choose $g(t, x, K, A)=$ $h(t, K) \delta_{x}(A)$ for $t \geq 0, x \in \mathbb{R}^{d}, K \in \mathcal{K}_{0}^{d}$, and measurable $A \subset \mathbb{R}^{d}$ we get

$$
\left.\mu(\psi, A)=\mathbf{1}\left\{\operatorname{card}\left(\psi_{W_{n}}\right) \geq 2\right\} \sum_{(x, K) \in \psi} h(R(\psi, x, K), K) \delta_{x}(A)\right), \quad \psi \in \mathbf{N}_{0}
$$

Recall that $V_{d}(\cdot)$ denotes the volume and assume, for instance, that $h(t, K)=V_{d}(t K)$ and a constant density $f \equiv 1$. Then we can use the hard-core property to deduce

$$
\int_{W_{n}} f\left(n^{-1 / d} x\right) \mu\left(\Psi_{W_{n}}, d x\right)=V_{d}\left(Z\left(\Psi_{W_{n}}\right)\right) \mathbf{1}\left\{\operatorname{card}\left(\Psi_{W_{n}}\right) \geq 2\right\},
$$

where $Z\left(\Psi_{W_{n}}\right)$ is the union of the grains of the growth-maximal hard-core model based on $\Psi_{W_{n}}$ as defined at (3.1). Similar considerations lead to a central limit statement for the surface area in the case of strictly convex particles.

\section{Acknowledgements}

The authors thank Prof. Daryl Daley for numerous discussions on different aspects of this work. Moreover the authors are grateful to the DFG (Deutsche Forschungsgemeinschaft) for partially funding this work via its research unit "Geometry and Physics of Random Spatial Systems".

\section{References}

[1] Andrienko, Y.A., Brilliantov, N.V. und Krapivsky, P.L. (1994). Pattern formation by growing droplets: the touch-and-stop model of growth. Journal of Statistical Physics $75,507-523$.

[2] Daley, D.J. and Last, G. (2005). Descending chains, lilypond growth protocol, and mutually nearest neighbour matching. Adv. in Appl. Probab. 37, 604-628.

[3] Daley, D.J., Mallows, C.L. and Shepp, L.A. (2000). A one-dimensional Poisson growth model with non-overlapping intervals. Stoch. Proc. Appl. 90, 223-241.

[4] Häggström, O. and Meester, R. (1996). Nearest neighbor and hard sphere models in continuum percolation. Random Structures Algorithms 9, 295-315.

[5] Heveling, M. and Last, G. (2006). Existence, uniqueness and algorithmic computation of general lilypond systems. Random Structures Algorithms 29, 338-350. 
[6] Hug, D. (1999). Measures, curvatures and currents in convex geometry. Habilitationsschrift, Albert-Ludwigs-Universität Freiburg.

[7] Kallenberg, O. (2002). Foundations of Modern Probability. Second Edition, Springer, New York.

[8] Last, G. (2010). Modern random measures: Palm theory and related models. In New Perspectives in Stochastic Geometry (eds. W. Kendall and I. Molchanov). Oxford University Press, Oxford.

[9] Last, G. and Penrose, M.D. (2012). Percolation and limit theory for the Poisson lilypond model. To appear in Random Structures $\&$ Algorithms.

[10] Penrose, M.D. (2007). Gaussian limits for random geometric measures. Electron. J. Probab. 12, 989-1035.

[11] Daley, D.J., Stoyan, D. and Stoyan, H. (1999). The volume fraction of a Poisson germ model with maximally non-overlapping spherical grains. Adv. Appl. Probab. 31, 610-624.

[12] Schneider, R. (1993). Convex Bodies: The Brunn-Minkowski Theory. Cambridge University Press. 